\title{
Low-Speed Fan Noise Attenuation from a Foam-Metal Liner
}

\author{
Daniel L. Sutliff* \\ NASA John H. Glenn Research Center at Lewis Field, Cleveland Ohio 44135 \\ and \\ Michael G. Jones ${ }^{\dagger}$ \\ NASA Langley Research Center Research, Hampton, Virginia 23681
}

DOI: $10.2514 / 1.41369$

\begin{abstract}
A foam-metal liner for attenuation of fan noise was developed for and tested on a low-speed fan. This type of liner represents a significant advance over traditional liners, due to the possibility of placement in close proximity to the rotor. An advantage of placing treatment in this region is that the acoustic near field is modified, thereby inhibiting the noise-generation mechanism. This can result in higher attenuation levels than could be achieved by liners located in the nacelle inlet. In addition, foam-metal liners could potentially replace the fan rub strip and containment components, ultimately reducing engine components and thus weight, which can result in a systematic increase in noise reduction and engine performance. Foam-metal liners have the potential to reduce fan noise by $4 \mathrm{~dB}$ based on this study.
\end{abstract}

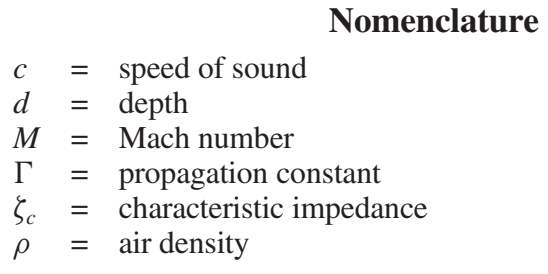

\section{Introduction}

$\mathbf{S}^{1}$ IGNIFICANT reduction in aircraft noise is required to meet ongoing noise regulation in the United States and Europe. Because the turbofan engine is a large contributor to aircraft noise, any attempt at overall reduction in aircraft noise must address engine noise [1]. A typical method is to attenuate the noise in the turbofan duct using acoustic liners. Standard liners with single-degree-offreedom (SDOF) perforate-over-honeycomb design are typically tuned to maximize attenuation at the blade-passage frequency. These liners have traditionally been installed in the inlet or exhaust nacelle, a relatively benign environment. It is desirable to install liners closer to the rotor, or even over the rotor, which is a much harsher pressure and temperature environment. If designed correctly, liners placed in this region can provide a pressure-release surface, mitigating the acoustic near field and thereby reducing the far-field noise emitted by the engine. This may result in more attenuation than can be achieved due to conventional liner mechanisms. Foam metal (Fig. 1) has the potential to survive in this environment. A liner made of foam metal and placed over the rotor has the potential to provide significant attenuation of fan noise.

This paper documents the acoustic attenuation characteristics of a foam-metal liner (FML) installed in a low-speed fan model. Foammetal intrinsic acoustic properties (characteristic impedance $\zeta_{c}$ and propagation constant $\Gamma$ ) measured via impedance-tube tests are

Presented as Paper 2897 at the 14th AIAA/CEAS Aeroacoustics Conference, Vancouver, Canada, 5-7 May 2008; received 2 October 2008; revision received 24 March 2009; accepted for publication 27 March 2009. This material is declared a work of the U.S. Government and is not subject to copyright protection in the United States. Copies of this paper may be made for personal or internal use, on condition that the copier pay the $\$ 10.00$ percopy fee to the Copyright Clearance Center, Inc., 222 Rosewood Drive, Danvers, MA 01923; include the code $\$ 10.00$ in correspondence with the CCC.

Q1 *Aerospace Engineer, Acoustics Branch, Mail Stop 54-3. Senior Member AIAA.

†Senior Research Scientist, Research and Technology Directorate, Structural Acoustics Branch. Associate Fellow AIAA. presented. Noise-reduction potential of a FML at standard inlet and over-the-rotor (OTR) locations were investigated. Flow data are presented, but due to the nature of the test rig, detailed effects on the fan performance parameters such as thrust and efficiency are not available.

\section{Impedance-Tube Testing}

\section{A. Test Samples}

Foam metal constructed from a cobalt alloy (see Fig. 2) was selected for this study because of the uniformity of its acoustic properties (i.e., constant acoustic properties throughout the material). Preliminary studies also indicate that this material is not readily flammable, does not readily absorb fluids, and can withstand expected mechanical loads. Samples with porosities of 20 to 100 pores per inch and densities (i.e., percentage of sample volume consisting of the cobalt alloy) of 6 to $8 \%$ were tested in the NASA Langley Research Center (LaRC) normal-incidence tube, and the two-thickness method [2] was used to determine the intrinsic properties of each foam type. The following sections describe the test methods used in this process.

\section{B. Test Procedures}

The experimental evaluation was conducted in three steps. First, the two-microphone method $[3,4]$ was used to measure the normalincidence acoustic impedance of two samples, composed of two and four 0.425 -in.-thick layers (the only thicknesses available) of foam metal, respectively. Next, the two-thickness method was used to educe intrinsic acoustic properties from these component measurements. Finally, the two-microphone method was used to measure the normal-incidence acoustic impedance of a third sample, composed of three 0.425 -in.-thick layers of foam, and the measured impedance spectra were compared with the corresponding impedance spectra predicted from these intrinsic acoustic properties.

\section{Two-Microphone Method}

The two-microphone method was used with the LaRC normalincidence tube (Fig. 3) to determine the acoustic impedance of each sample. These data were generated with a random noise acoustic source at overall sound pressure levels [(OASPL), integrated over a frequency range of 500 to $3000 \mathrm{~Hz}$ ] of 120 and $140 \mathrm{~dB}$, as measured by the reference microphone flush-mounted $0.25 \mathrm{in}$. from the surface of the sample. Data were acquired at frequencies from 500 to $3000 \mathrm{~Hz}$, in increments of $25 \mathrm{~Hz}$. In total, eight tests were conducted for this investigation (two source levels, four samples). As expected, results acquired with each sample were observed to be independent 


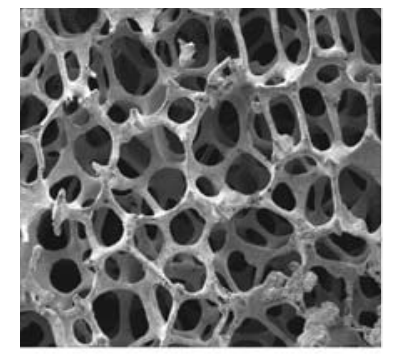

Desirable foam-metal characteristics:

- excellent acoustic absorption

- high temperature capability

- high impact resistance

- resistance to fluid absorption

Image above is $17 \mathrm{x} 17 \mathrm{~mm}$

Fig. 1 Close-up of foam metal (60 ppi, $8 \%$ density).

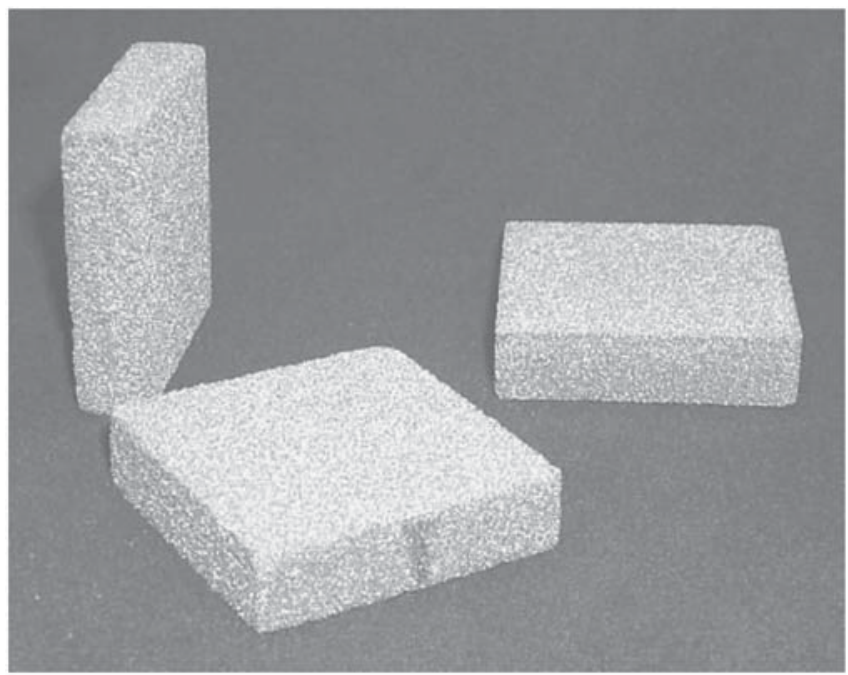

Fig. 2 Photograph of foam-metal samples $(2 \times 2 \times 0.425 \mathrm{in}$.$) .$

of the source OASPL over the range of OASPLs used in this study. This was also observed to be true for the foams considered in this study. Thus, for the sake of brevity, only the results for an OASPL of $140 \mathrm{~dB}$ are presented in this report.

\section{Two-Thickness Method}

The two-thickness method is well established for educing the intrinsic properties (characteristic impedance $\zeta_{c}$ and propagation constant $\Gamma$ ) of bulk-absorbing structures. The core of this method is the solution of the following two equations:

$$
\begin{aligned}
& \zeta_{1}=\zeta_{c} \operatorname{coth}\left(\Gamma d_{1}\right) \\
& \zeta_{2}=\zeta_{c} \operatorname{coth}\left(\Gamma d_{2}\right)
\end{aligned}
$$

where $\zeta_{1}$ and $\zeta_{2}$ represent measured impedances on two separate test samples of depths $d_{1}$ and $d_{2}$, taken from what is assumed to be a homogeneous continuous structure. If the sample depths are selected such that $d_{2}=2 d_{1}$, the characteristic impedance and propagation constant spectra can be determined as follows:

$$
\begin{gathered}
\zeta_{c}=\sqrt{\zeta_{1}\left(2 \zeta 2-\zeta_{1}\right)} \\
\Gamma=\Gamma_{r}+I \Gamma_{i}=\frac{1}{2 d_{1}} \log \left(\frac{1+a}{1-a}\right) \\
a=\sqrt{\frac{2 \zeta_{2}-\zeta_{1}}{\zeta_{1}}}
\end{gathered}
$$

The impedance $\zeta_{s}$ of a third sample with thickness $d_{s}$ can then be determined using

$$
\zeta_{s}=\zeta_{c} \operatorname{coth}\left(\Gamma d_{s}\right)
$$

A comparison between the measured impedance of the third sample and that predicted via Eq. (6) can be used to assess the validity of the educed parameters. The $L 2$-norm, $L^{2}$, is a vector norm for complex quantities that can be conveniently used for this evaluation. It is computed as follows:

$$
L 2=\frac{1}{N} \sqrt{\sum_{i=1}^{N}\left|\zeta_{m, i}-\zeta_{p, i}\right|^{2}}
$$

where $\zeta_{m, i}$ and $\zeta_{p, i}$ are the measured and predicted impedances, respectively, at the $i$ th frequency, and $N$ is the total number of frequencies used in the evaluation. Ideally, the value of $L 2$ should be zero, indicating exact comparison between the measured and predicted impedance spectra for samples with depths that are different from those used as input to the method. Thus, any departure from zero is 1) a measure of experimental error or 2) a breakdown of the continuum assumption (e.g., material imperfections).

\section{Results}

The measured impedance spectra for 0.85 - and 1.70-in.-thick samples (two and four layers of foam metal) are provided in Fig. 4. Equations (3-5) were then used to compute the corresponding characteristic impedance and propagation constant for this foam metal. These intrinsic acoustic properties were then used to predict the acoustic impedance spectrum for the 1.275-in.-thick sample. A comparison of the predicted and measured acoustic impedance spectra is provided in Fig. 5. The comparison is exceptional, indicating that the intrinsic properties of the foam metal have been successfully educed. The $L 2$-norm, $L^{2}$, computed from the comparison of the predicted and measured acoustic impedance spectra for this sample has a value of 0.21 . For the frequencies used in this study ( $25 \mathrm{~Hz}$ increments from 400 to $3000 \mathrm{~Hz}$ ), this corresponds to an average error between the measured and predicted resistances and reactances (real and imaginary components of acoustic impedance) of $0.015 \rho c$ ( $\rho c$ is the characteristic impedance of air). This extremely small error provides confidence in the ability of the model to predict the normal-incidence acoustic impedance spectra that would be measured for any sample thickness within reasonable proximity to those included in the current study.

These impedances were then used to predict the absorption coefficient spectra for 1- and 2-in.-thick samples of this foam type

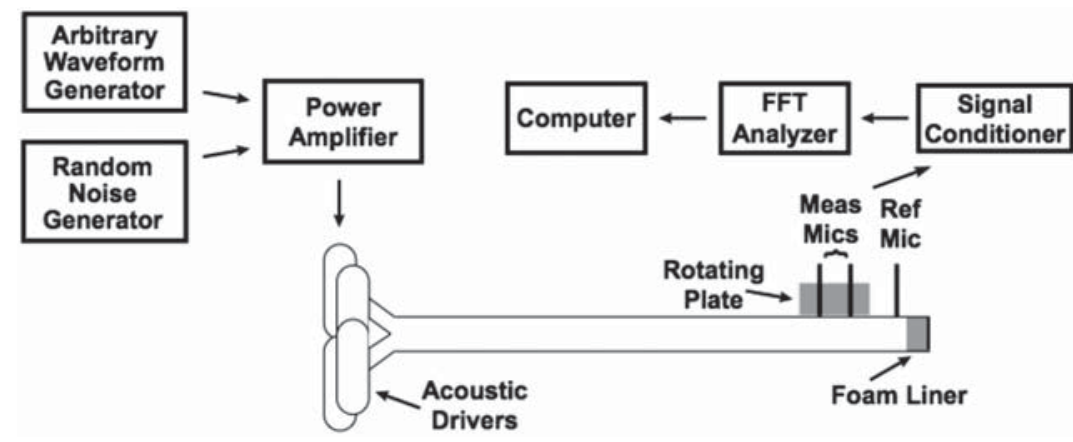

Fig. 3 Sketch of LaRC normal-incidence tube with supporting instrumentation. 


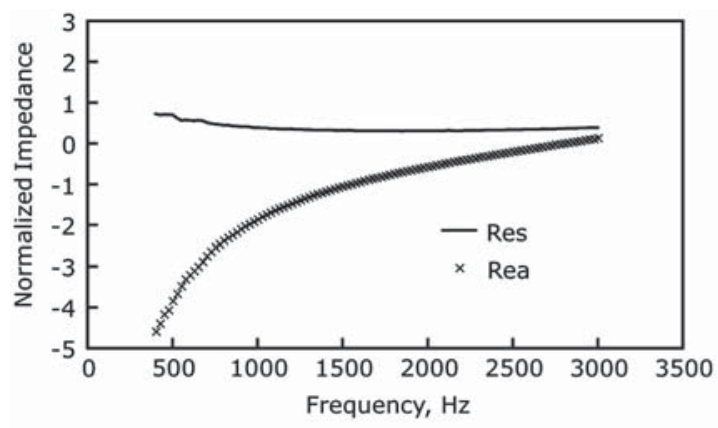

a) 0.85 in. thick

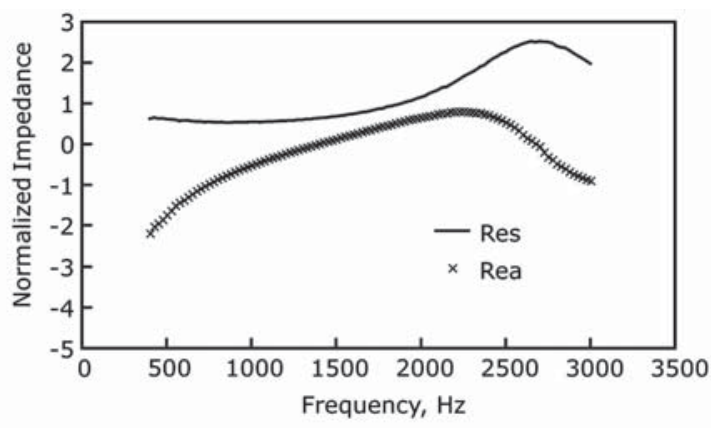

b) $1.70 \mathrm{in}$. thick

Fig. 4 Normal incidence acoustic impedance for foam-metal samples.

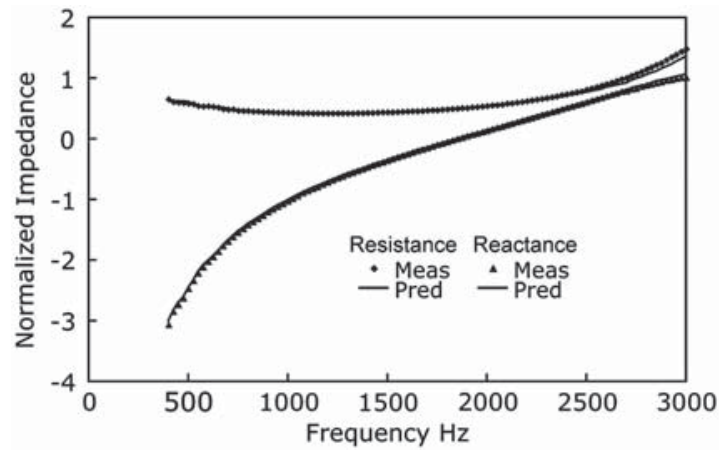

Fig. 5 Comparison of predicted and measured acoustic impedance spectra for 1.275-in.-thick sample.

(Fig. 6). Based on these results, the 80-pores-per-inch, 8\%-density cobalt alloy was deemed suitable for further detailed evaluation.

\section{Low-Speed-Fan Testing}

\section{A. Advanced Noise-Control-Fan Test Bed}

The test bed for the FML was the advanced noise-control fan [5] (ANCF), a 4-ft-diam low-speed fan used for validation of noisereduction concepts. The ANCF is a highly configurable, ducted fan rig located in the Aero-Acoustic Propulsion Laboratory [6] (AAPL) at the NASA John H. Glenn Research Center at Lewis Field. The ANCF, shown in Fig. 7, operates inside an enclosed compact anechoic far-field arena within the AAPL. The AAPL is a hemispherical anechoic (to $125 \mathrm{~Hz}$.) test facility that allows for far-field noise measurements. An exterior view of the 65 -ft-high dome is shown in Fig. 8.

The nominal operating condition of the ANCF is $1800 \mathrm{rpm}$ (375 ft/s tip speed), providing an inlet duct Mach number of $\sim 0.15$ and a fundamental blade passing frequency (BPF) of $\sim 500 \mathrm{~Hz}$. The ANCF is composed of a series of 11- or 12-in.-long cylindrical spools that are axially interchangeable, enabling rapid testing of a variety of configurations. The ANCF is a very-low-pressure rise fan and, as

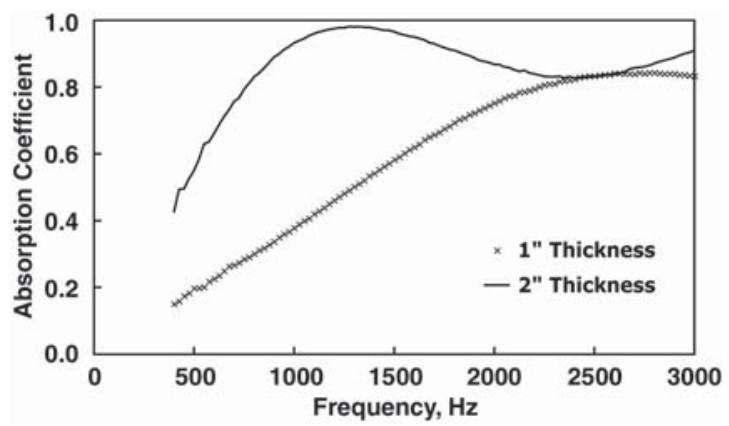

Fig. 6 Absorption coefficient spectra for two thicknesses of $80 \mathrm{ppi}, 8 \%$ density foam metal.

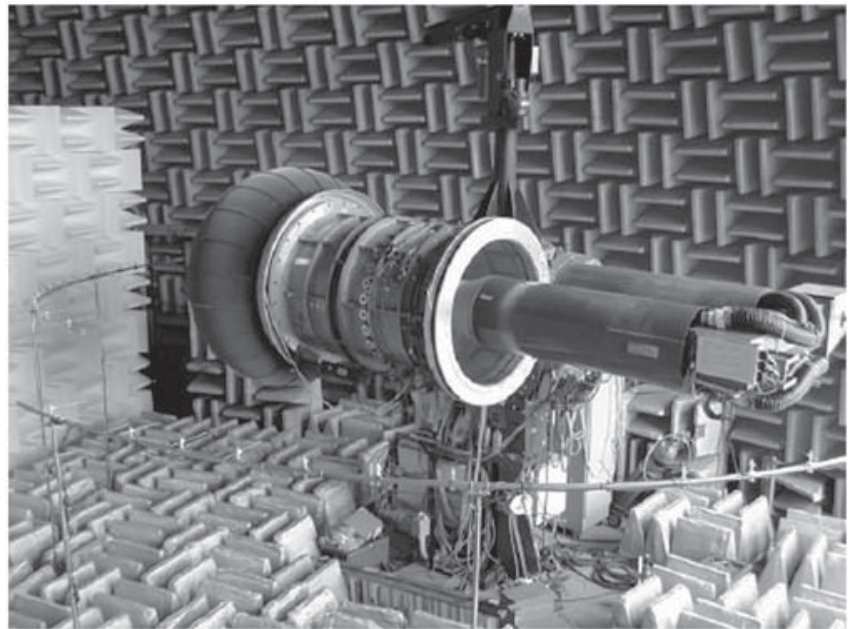

Fig. 7 Advanced noise-control fan.

such, traditional performance parameters such as thrust and fan efficiency are not readily measurable. The performance impact of a given noise-reduction concept is deduced by comparing basic flow measurements such as steady pressure and/or velocity behind the rotor.

\section{B. Test Hardware and Description}

Based on the impedance-tube test results of the foam-metal samples and the known acoustic characteristics of the ANCF, a 9-in.long axial liner, with a total depth of 2 in. and with foam-metal characteristics of $80 \mathrm{ppi}$ and $8 \%$ density, was designed and integrated

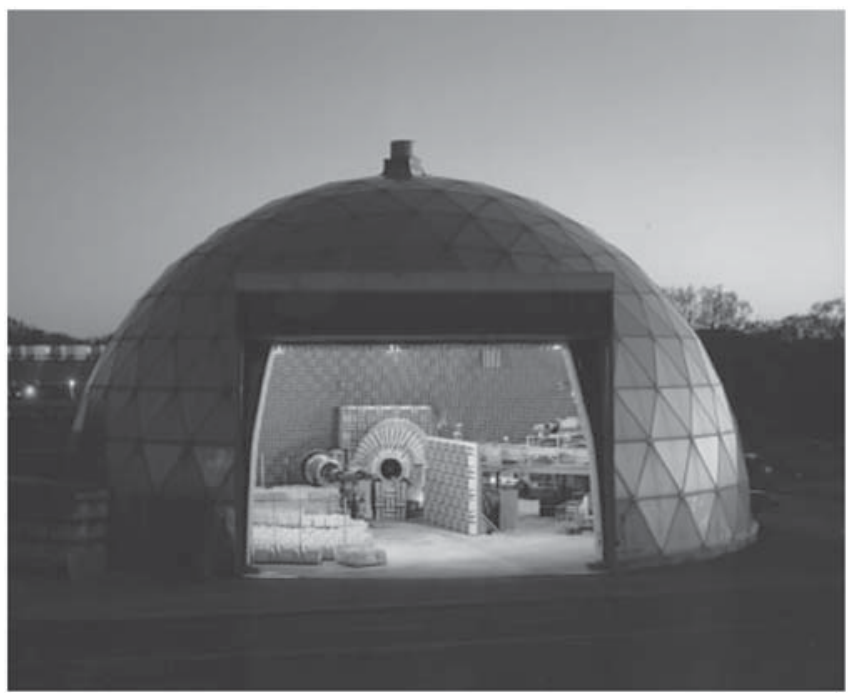

Fig. 8 Aero-Acoustic Propulsion Laboratory. 


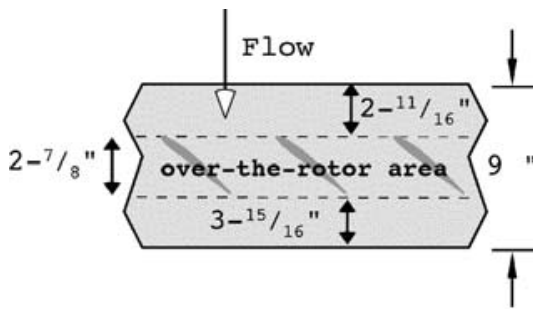

a) Top view

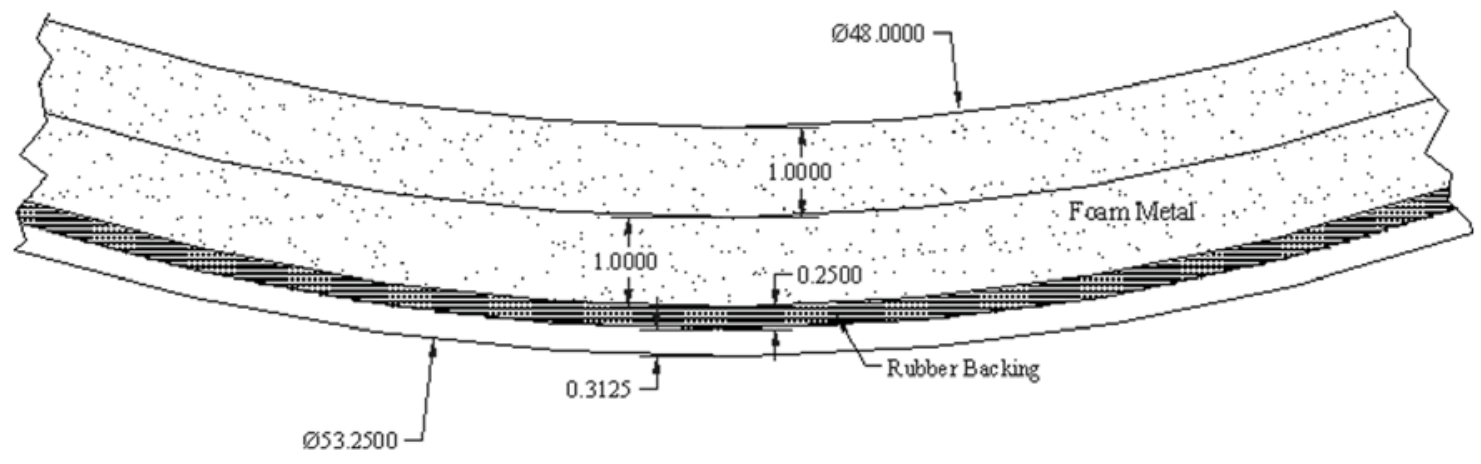

b) Side view

Fig. 9 FML schematics.

into a 12 in. spool piece. The FML was manufactured in segments: two 1 in. layers, each consisting of one-eighth arc of the circumference. Figure 9 shows top- and side-view schematics of the FML design. Figure 10 shows the FML spool piece during model buildup, and Fig. 11 shows the FML installed in the OTR position.

The foam-metal liner was tested in several axial locations. The schematics of the configurations tested are presented in Fig. 12. Two traditional inlet locations (Fig. 12a) and the OTR (Fig. 12b) and overthe-stator (OTS) (Fig. 12c) configurations were tested. When the FML spool was installed over the rotor, it was situated so that the projected rotor path was approximately centered over the exposed liner material. The OTR configurations were tested without stator vanes installed (rotor only), to isolate the source. The other liner configurations tested had 14 stator vanes installed behind the rotor.

A deliberate choice was made to perform all the OTR testing without stators, due to mechanical limitations, and to investigate the effect of FML/OTR on the rotor source alone. Obtaining insertion losses for the treatment on the source for which it was designed was the primary goal. Stators behind the rotor would mask the OTR attenuation of the rotor noise.

The effect of depth of the OTR FML was also investigated. Because the FML was built with two 1 in. layers, applying hard-wall

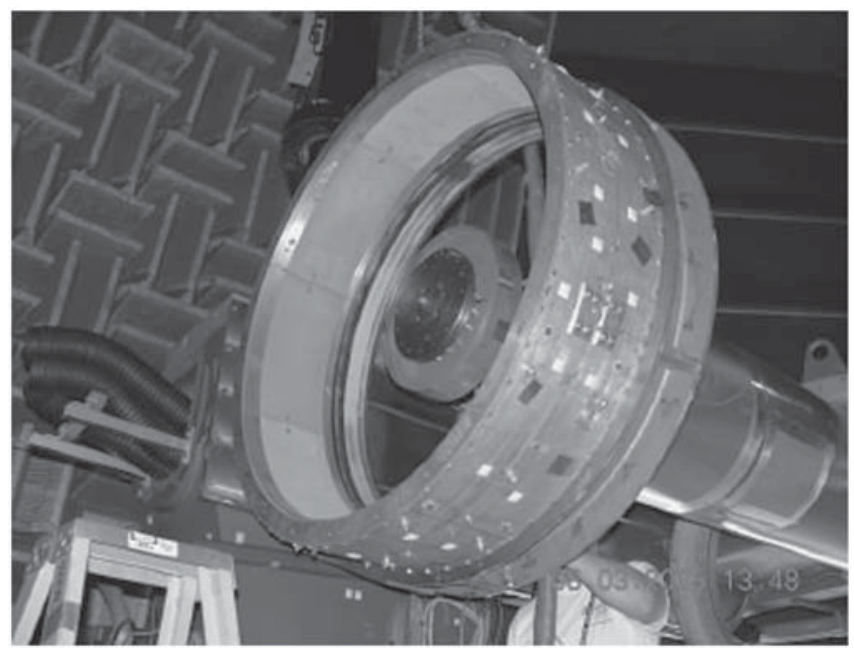

Fig. 10 FML spool piece. tape between the liner segments layers effectively reduced the liner depth. Thus, two liner depths, 1 and 2 in., were tested. The primary configurations were run with a rotor tip gap of $3 / 32 \mathrm{in}$. (1.8\% relative to a rotor tip chord of $5.25 \mathrm{in}$.). The liner spool was modified to obtain a rotor tip gap of $1 / 32$ in. ( $0.6 \%$ of rotor tip chord) to investigate the interaction between the rotor tip gap and foam metal.

SDOF liners from an earlier program [7,8], which were designed to target approximately the same acoustic characteristics, were used as comparative baselines. The acoustic performance achieved with the FML was compared with those previously measured with two standard SDOF liners. The normalized design resistances for these liners were 1.7 and 1.0 (impedance components normalized by $\rho c$ ), respectively. The liner core depths were 0.85 and 1.0 in., resulting in resonance frequencies of 3221 and $2872 \mathrm{~Hz}$, respectively. The highresistance liner was installed in the inlet, and the low-resistance liners (an annular set) were installed in the exhaust (Fig. 12d).

Finally, using tape to convert sections of the OTR FML to the hard wall localized the attenuation. In addition to this, each configuration tested then had the liner taped over to create a hard-wall configuration to provide the baseline for that configuration.

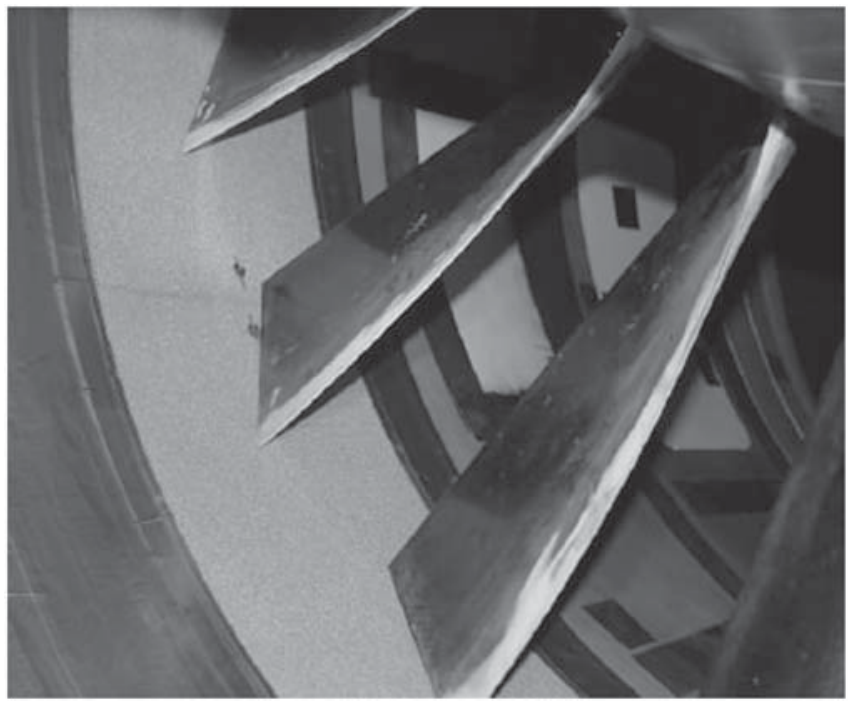

Fig. 11 FML spool installed over the rotor. 


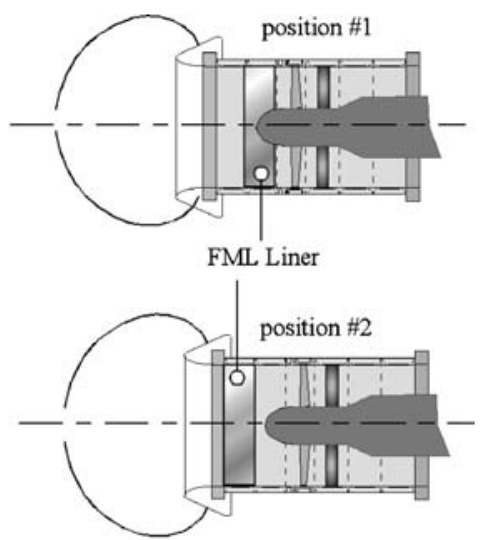

a) FML in 2 inlet locations

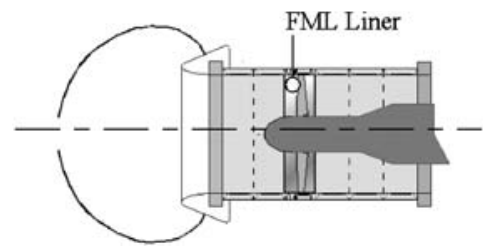

b) FML over the rotor (1" \& 2" depth)

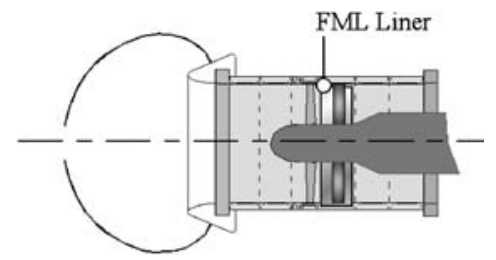

c) FML over the stator

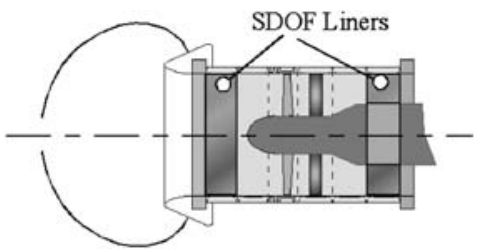

d) SDOF liner in inlet and exhaust duct

Fig. 12 Schematic of liner configurations tested on ANCF.

Far-field acoustic directivity, total and static pressure behind the rotor, steady-state and dynamic wall pressures, and 2-component

Q2 hot-film data were acquired. Table 1 indicates the data acquired for each configuration. Table 2 shows the corrected rpm at which each type of data was acquired.

Far-field acoustic directivities were acquired using 30 microphones placed at a $12 \mathrm{ft}$ radius from the duct centerline. Fifteen of these were in an arc centered about the inlet exit plane (0-90 deg measured from the inlet axis) and 15 were centered about the exhaust exit plane (90-135 deg, with $180 \mathrm{deg}$ being the exhaust axis). The spectra of each time history are processed to analyze the broadband content by removing the tones generated by the fan (shaft orders and harmonics) and integrating about a fan harmonic. This can be done exactly, because the data are acquired synchronously to the shaft rotation. For example, the first harmonic band is defined as the integration from $0.5 B$ to $1.5 B$, the second harmonic band from $1.5 B$ to $2.5 B$, etc. $B$ is the number of blades, and so the first harmonic band is centered on 16 shaft orders and is the integration from 8 to 24 shaft orders (Fig. 13). The data can then be converted to power by squaring the pressure and multiplying by the appropriate area, then

Q3 normalizing by specific acoustic impedance (PWL). All acoustic data presented in this paper are broadband as defined by this process.

\section{Results from Low-Speed-Fan Testing}

\section{Acoustic Data}

Data were first acquired with the 2 in. depth FML installed in the inlet duct. Two configurations were tested: position 1, with the spool piece closest to the fan, and position 2, with the spool piece closest to the inlet lip (refer to Fig. 12a). Broadband spectra from two representative microphones, one from the forward arc and one from the aft arc, are presented in Fig. 14, comparing spectra from the inlet FML configurations with those obtained with a hard-wall configuration. A clear acoustic attenuation from shaft order 16 (BPF) to 48 of up to $3 \mathrm{~dB}$ is seen at the forward arc microphone (Fig. 14a). This matches the predicted absorption reasonably well. Note that with the liner in the inlet, no change in the aft-radiated spectra is seen (Fig. 14b).

The directivity of the broadband-radiated noise from these inlet configurations is shown in Fig. 15. The first harmonic band shows limited attenuation, as expected, because the liner was not designed to attenuate that frequency range. The attenuation in the forward arc (0-90 deg) is seen most notably in the second through fourth harmonic bands (Figs. 15b-15d), which is the design frequency (refer to back to Fig. 6). Slightly greater attenuation with the FML in position 2 can be seen in these harmonic bands. The attenuation in the

Table 2 Fan rpm range of data acquired

\begin{tabular}{lc}
\hline \hline Type & Corrected rpm \\
\hline Far-field acoustic & $1800,1600,1400$ \\
Unsteady/steady wall pressures & $1800,1600,1400$ \\
Total/static pressure traverses & 1800 \\
Hot-film traverses & 1800 \\
\hline \hline
\end{tabular}

Table 1 Data acquired for each configuration tested

\begin{tabular}{lccccc}
\hline \hline Type of data & Hard wall & OTR FML: 1 in. & OTR FML: 2 in. & SDOF & Inlets/OTS \\
\hline & & 3/32 tip gap & & & \\
Far-field acoustic & Yes & Yes & Yes & Yes & Yes \\
Unsteady/steady wall pressures & Yes & No & Yes & No & No \\
Total/static pressure traverses & Yes & No & Yes & No & No \\
Hot-film traverses & Yes & No & Yes & No & No \\
& & 1/32 tip gap & No & No & Yes \\
Far-field acoustic & Yes & Yes & No & No & No \\
Unsteady/steady wall pressures & Yes & Yes & No & No & No \\
Total/static pressure traverses & Yes & Yes & No & No & No \\
Hot-film traverses & Yes & Yes & &
\end{tabular}




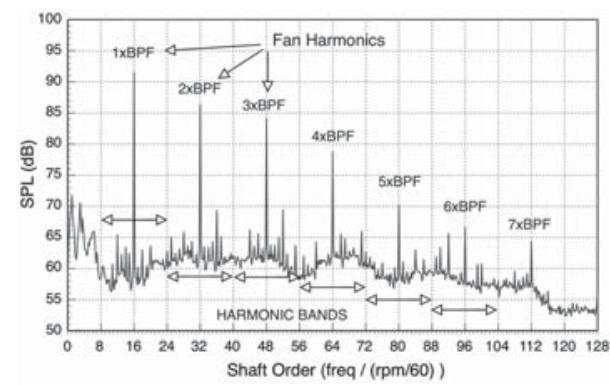

a) Original spectral sample

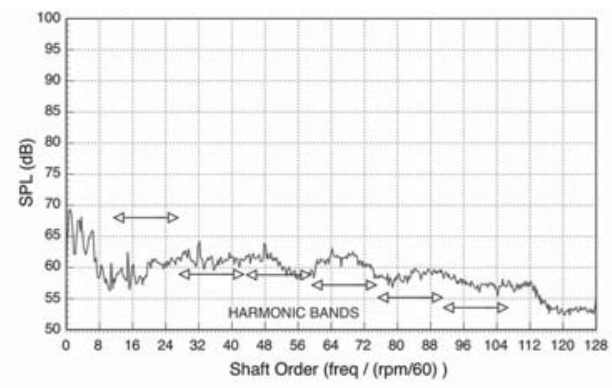

b) Spectral sample with fan harmonics removed

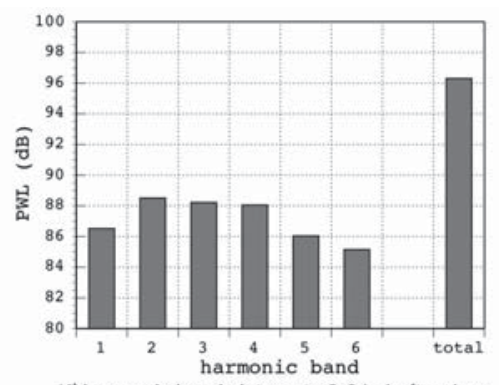

$1^{\text {st }}$ harmonic band : integrate $8-24$ shaft orders $2^{\text {nd }}$ harmonic band : integrate $24-40$ shaft orders $3^{\text {rd }}$ harmonic band : integrate $40-56$ shaft orders etc.

Fig. 13 Example of far-field data reduction into harmonic bands.

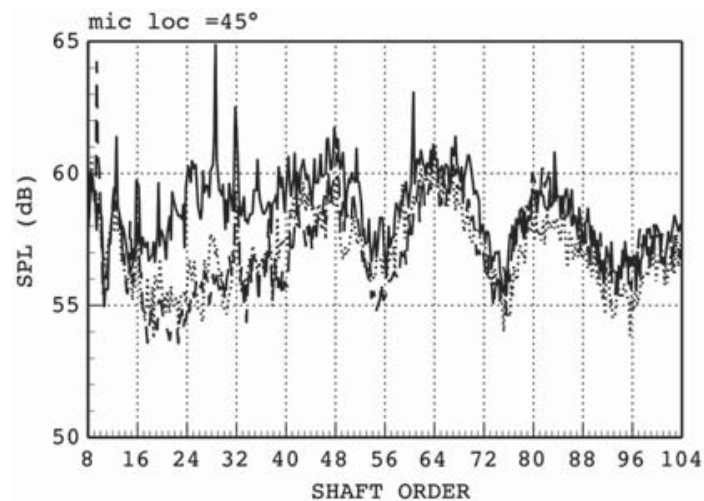

a) Spectra from mic at $\mathbf{4 5} \mathrm{deg}$

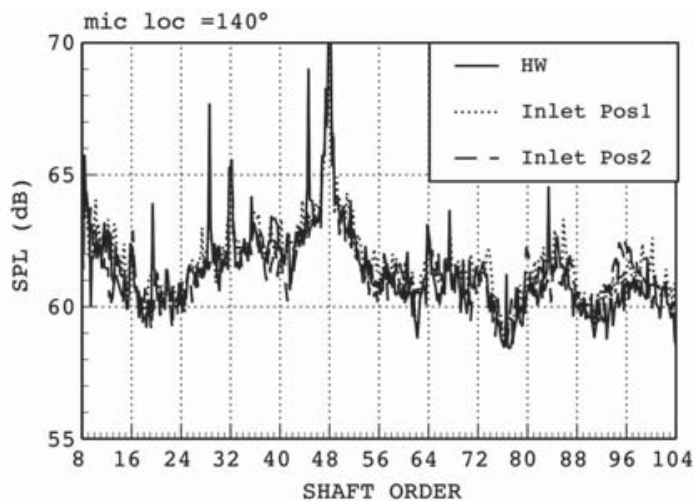

b) Spectra from mic at $140 \mathrm{deg}$

Fig. 14 Representative fan spectra with FML installed in two inlet locations compared with the hard wall.

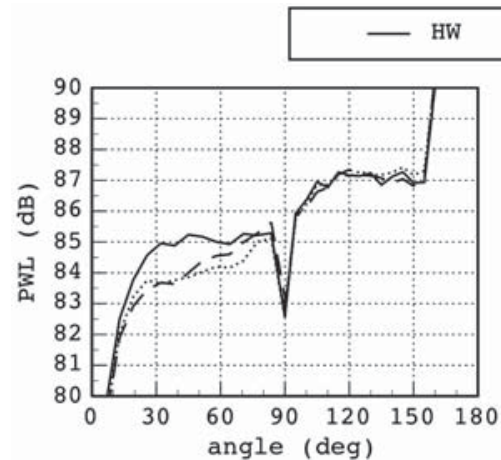

a) First harmonic band

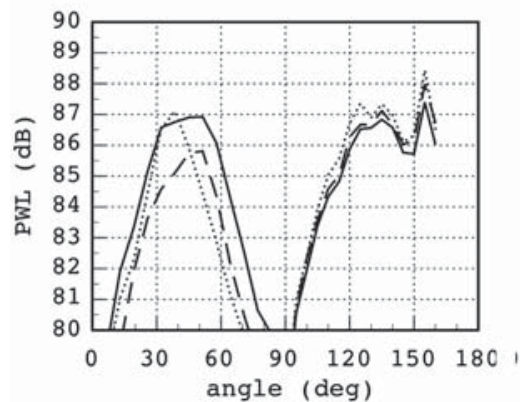

d) Fourth harmonic band

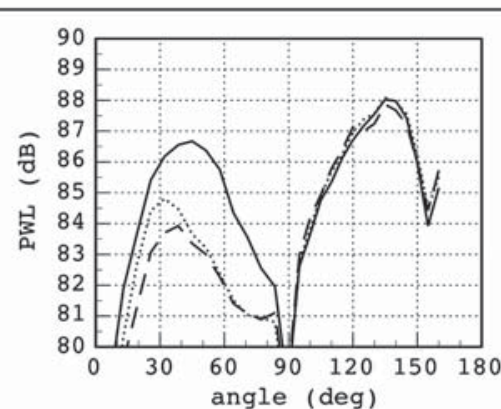

b) Second harmonic band

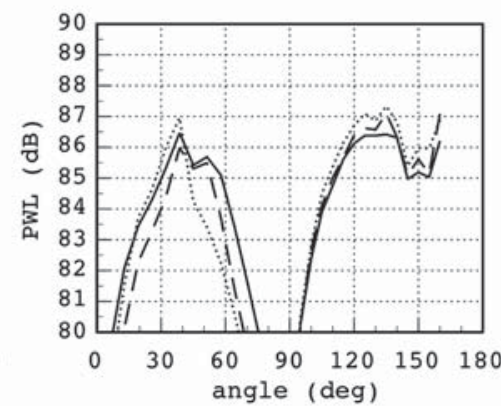

e) Fifth harmonic band c) Harmonics bands 
higher harmonic bands (Figs. 15e and 15f) is very modest. The radiated levels in the aft arc (90-165 deg) do not change significantly in the first through fifth harmonic bands. A small increase is noted in the sixth harmonic band. It is speculated that this is due to increased turbulence from the higher surface roughness of the FML being ingested by the rotor.

The spectral character of the FML installed over the rotor compared with the hard wall is shown in Fig. 16; the broadband directivity for these configurations is shown in Fig. 17. (As the ANCF is rearranged to create this configuration, a new hard-wall configuration is created by using hard-wall tape to cover the liner, and then tested.) Significantly greater attenuation (compared with the inlet positions), up to $5 \mathrm{~dB}$, over the shaft order range above 16 (BPF) is measured in both the forward and aft far-field arcs. This is due to the anticipated near-field modification resulting from placing the treatment in close proximity to the source. It is not clear from the farfield acoustic data if this is a result of increased acoustic attenuation from the rotor sou rce, or due to a modification of the source itself (possibly from aerodynamic effects in the fan tip region), or a combination of effects.
Figure 18 shows the broadband attenuation in each harmonic band (relative to the hard-wall configuration) obtained with FML configurations and that obtained with a traditional SDOF liner installed in the inlet duct and inner and outer walls of the exhaust duct (simultaneously). Figure 18a is the attenuation obtained from the FML in the inlet configurations (see Fig. 12a), Fig. 18b is the attenuation obtained from the FML in the OTR configuration (see Fig. 12b), and Fig. 18c is the attenuation obtained from SDOF liner configuration (see Fig. 12d). The FML liner provides a 9 in. length of treatment, and the 3 SDOF liners each provide an $18 \mathrm{in}$. axial length of treatment. Note that the sources are different in each configuration, and hence the comparison is based on insertion loss. (It is unlikely that the insertion loss for the SDOF liners would change if there were no stators present.)

A comparison of Figs. 18a and 18b shows that placing the FML over the rotor results in more attenuation in the inlet arc than occurred in the inlet FML configurations. In addition, attenuation was achieved in the aft arc that was not present with the FML located in the inlet. This illustrates the acoustic benefits obtained from liner placement at the source.

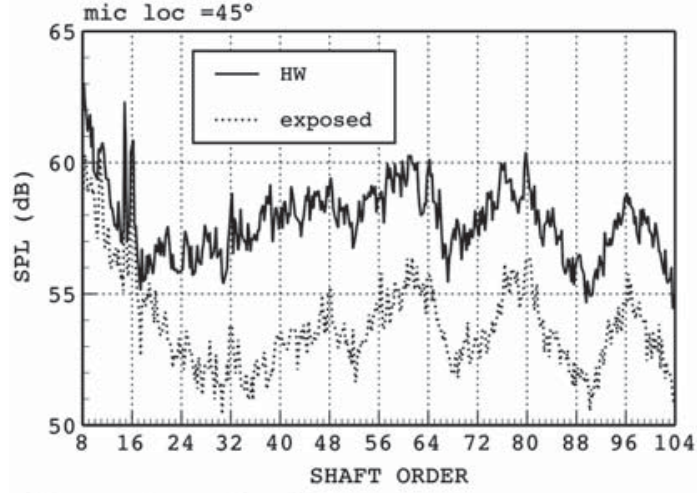

a) Spectra from mic at $\mathbf{4 5} \mathrm{deg}$

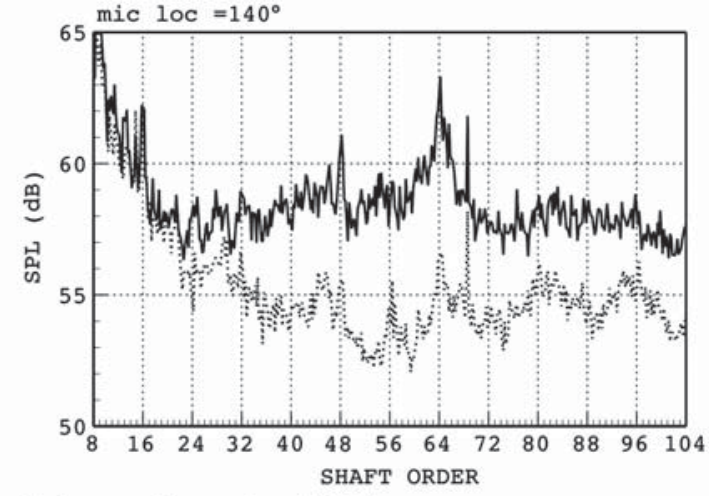

b) Spectra from mic at $120 \mathrm{deg}$

Fig. 16 Representative fan spectra with FML installed over the rotor compared with the hard wall.

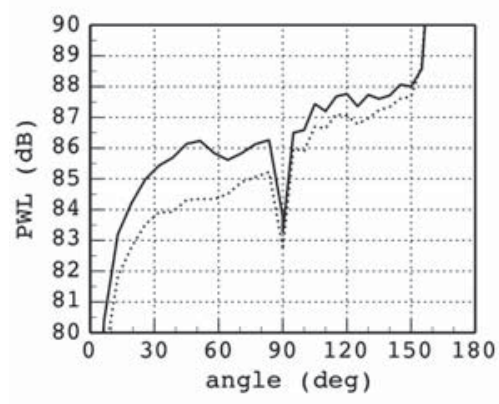

a) First harmonic band

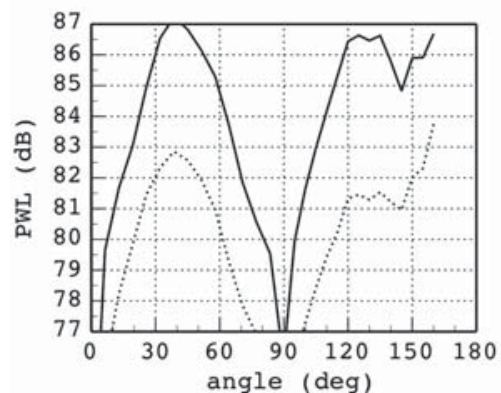

d) Fourth harmonic band

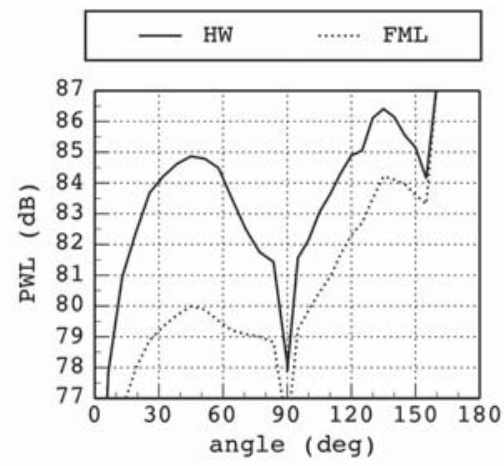

b) Second harmonic band

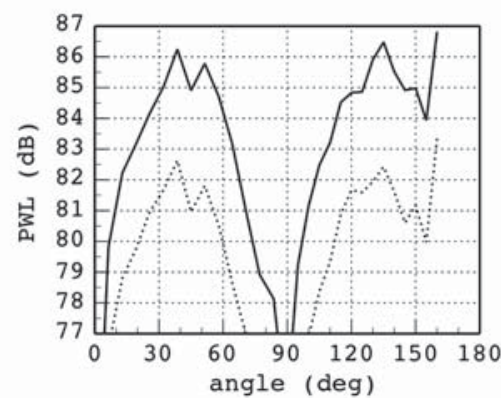

e) Fifth harmonic band

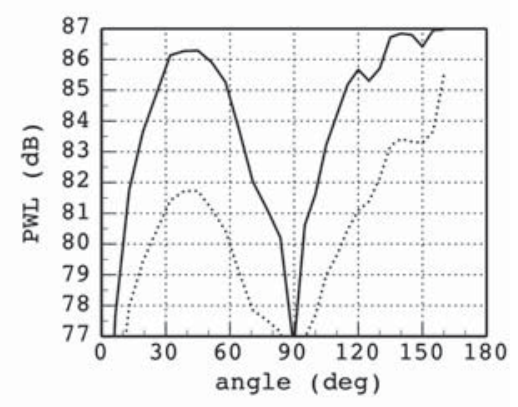

c) Third harmonic band

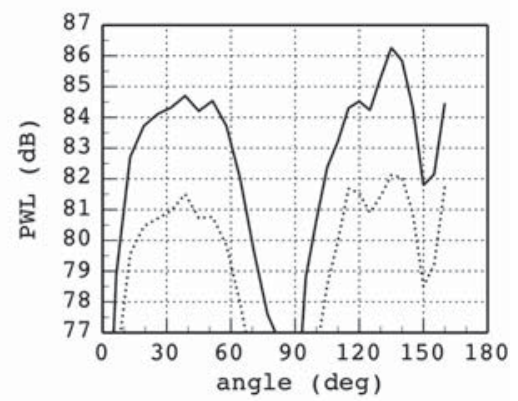

f) Sixth harmonic band

Q11 Fig. 17 Far-field directivity of fan with FML installed over the rotor compared with hard wall (HW). 


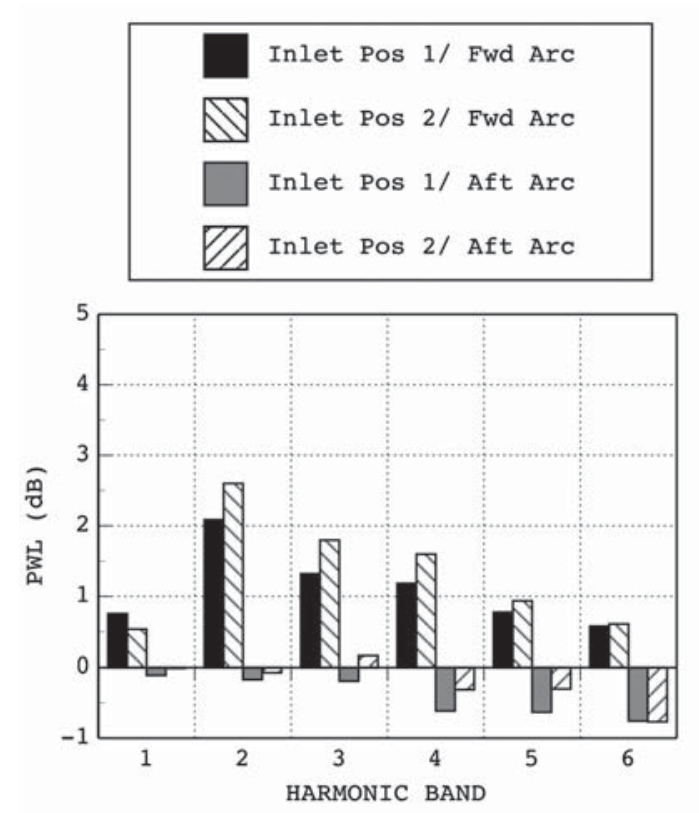

a) FML in inlet

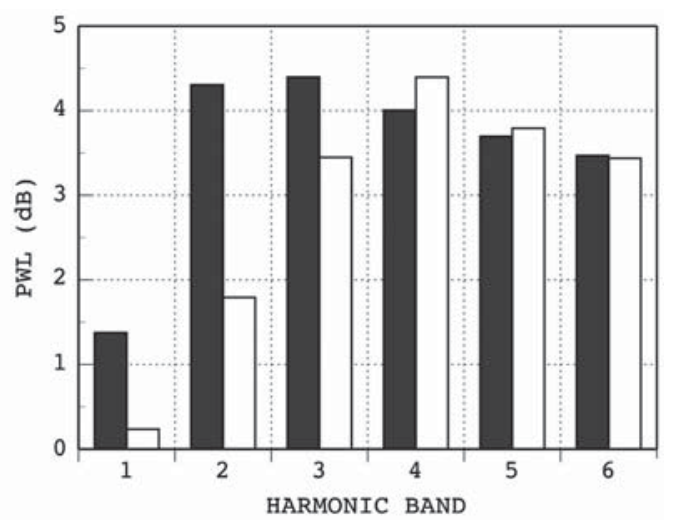

b) FML over rotor

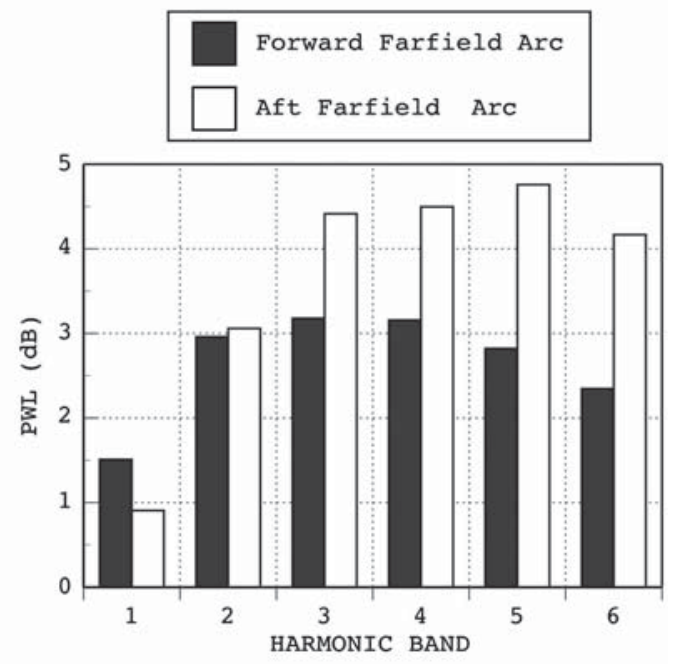

c) SDOF in inlet/exhast

Fig. 18 Far-field attenuation relative to the hard wall for FML compared with SDOF liners.

The attenuation obtained from the 9-in.-long FML is equal to that obtained with a combined 54 in. length of SDOF liners, as can be seen in the comparison between Figs. $18 \mathrm{~b}$ and $18 \mathrm{c}$. This creates the potential for additional attenuation by combining liner types or reducing weight by removing the need for inlet or exhaust duct length required for standard treatment.

To determine the efficacy of the treatment location relative to the rotor, hard-wall tape was used to cover one or more of three physical sections of the OTR liner. Figure 19 provides the dimensions of each section of the FML that could be taped to effectively change the length of the liner. To first order, this effectively shortened the liner and/or changed its axial location relative to the projected rotor path. In reality, axial acoustic propagation occurs beneath the taped surface, but is greatly inhibited due to absorption within the foam metal. Thus, although this is not an exact representation of such a liner change due to the axial communication path within the liner, it

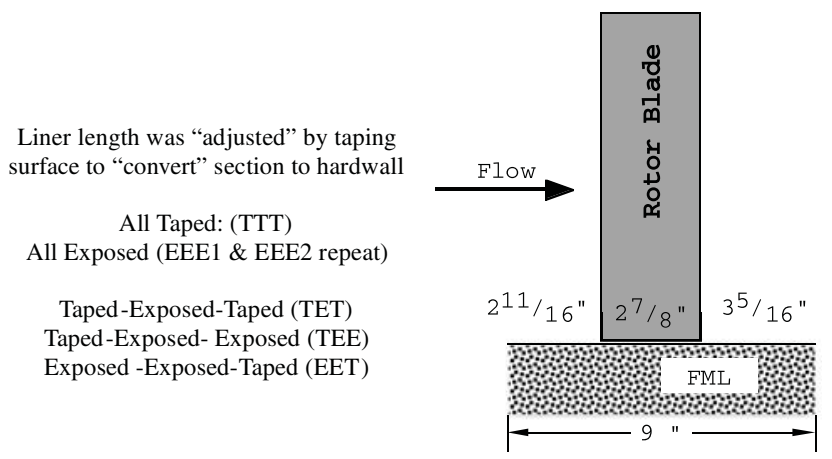

Fig. 19 Close-up schematic showing sections of FML. does provide an indication of what would be achieved if the taped portion were replaced with a completely solid axial segment.

The far-field attenuation achieved in the forward and aft arcs for these alternative configurations is shown in Fig. 20. Considering the forward arc (Fig. 20a), the forward two-thirds of the liner achieves all of the reduction of the full liner (EET vs EEE). Though it was not tested, by examining the attenuation achieved from the section immediately over the rotor (TET) and noting that most of the attenuation is lost when the first section of the liner (TEE) is removed, it is inferred that the majority of attenuation is a result of the forward one-third of the liner. This linear analysis is not necessarily definitive in this region, and this inference must be used with caution. Nonetheless, it appears that the attenuation in the forward arc is a result of the section of the liner just in front of the rotor. Further, this suggests the mechanism may be primarily acoustic attenuation rather than aerodynamic source modification.

Similar analysis of the attenuation achieved in the aft arc indicates that the majority of the attenuation achieved is a result of the section immediately over the rotor (TET). Adding the first section (EET) provides an insignificant increase in attenuation; adding the third, or aft, section increases the attenuation by about $1 \mathrm{~dB}$. This may support a combined acoustic attenuation/source modification mechanism.

The effect of depth of the OTR FML was also investigated. Because the FML was built with two 1 in. layers (recall Fig. 9b), the depth was reduced by applying hard-wall tape between the liner segments layers. Thus, two liner depths, 1 and 2 in. were tested. Also, the prior configurations were all run with a rotor tip gap of 3/32 in. (1.8\% relative to rotor tip chord of 5.25 in.). The liner spool was modified to obtain a rotor tip gap of $1 / 32$ in. ( $0.6 \%$ of rotor tip chord).

Figure 21 shows the attenuation achieved from these configurations relative to a hard-wall baseline (again, a new hardwall baseline at $1 / 32$ in. tip gap was tested.) Decreasing the liner 

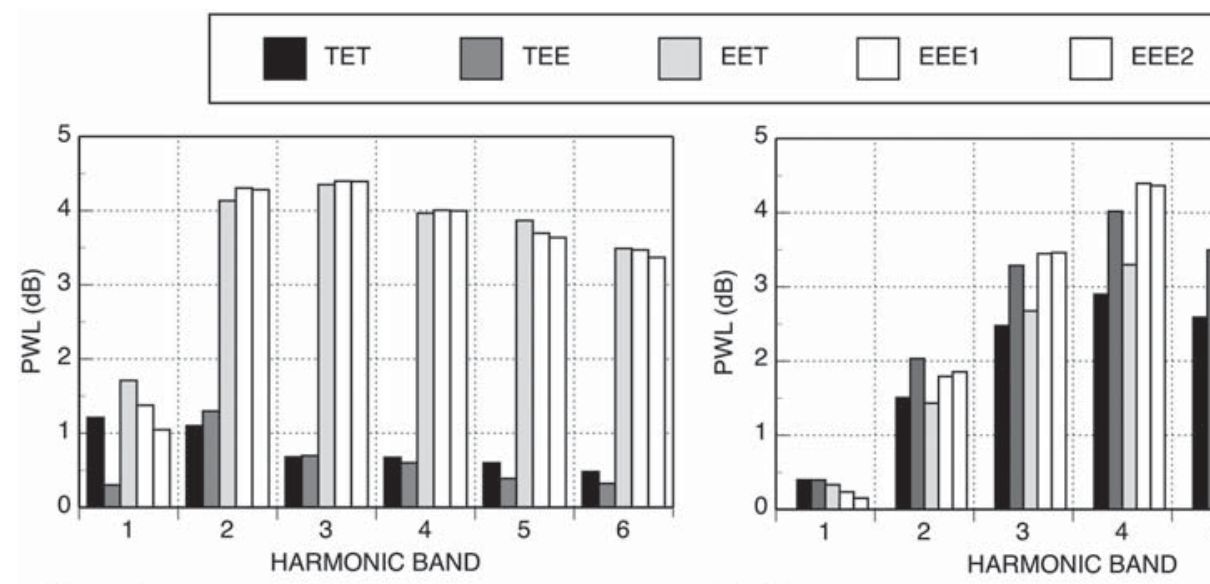

a) Forward arc

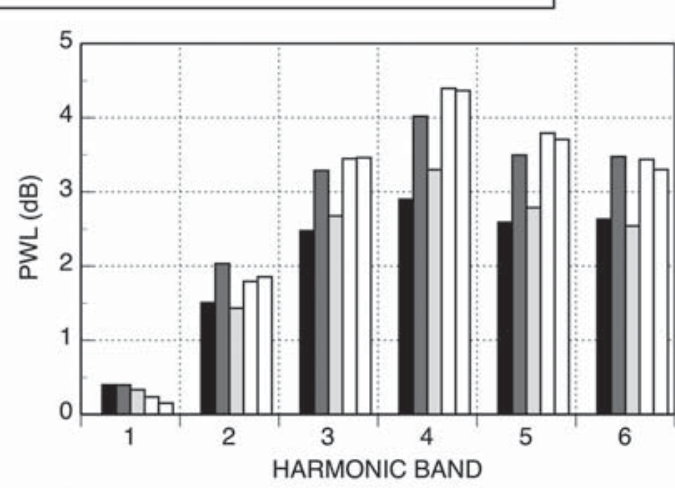

Q12 Fig. 20 Attenuation achieved with FML OTR sections: T denotes taped and E denotes exposed.

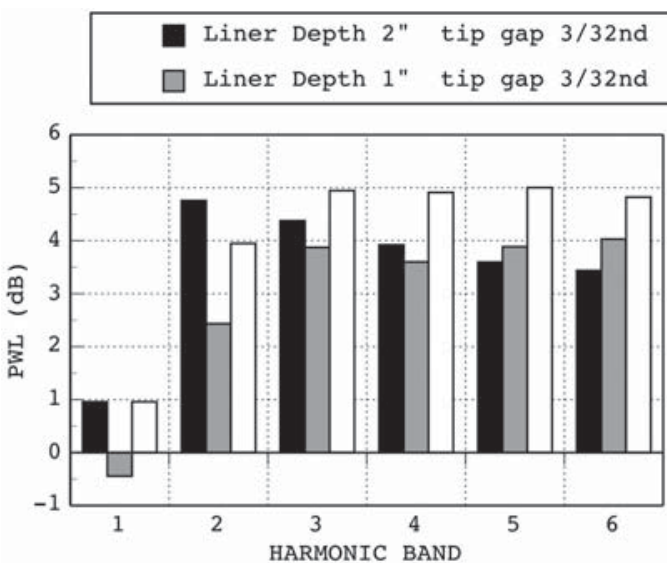

a) Forward arc

b) Aft arc

b) Aft are

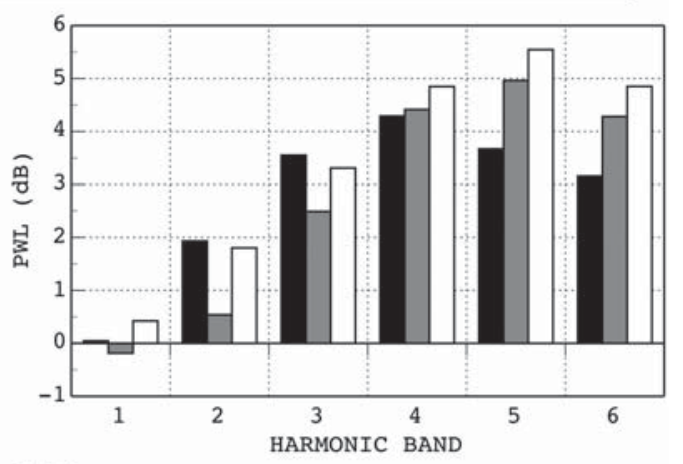

Fig. 21 Effect of FML depth and rotor tip gap on attenuation.

depth had a detrimental impact on the attenuation in the lower frequencies (harmonic bands 1-3), but increased the attenuation at the higher frequencies (harmonic bands 5-6), as might be expected from a simplistic wavelength analysis that assumes deeper liners are tuned to longer wavelengths. Reducing the tip gap (with a $1 \mathrm{in.} \mathrm{liner)}$ had an overall positive impact on the attenuation. It may be that the tip vortex is more influenced by the smaller tip gap, and hence closer porous surface, implying that the greater attenuation is at least partially a result of source modification.

The FML spool was installed over the stators (OTS-Fig. 12c) to determine the effect in a nonrotating region. Figure 22 shows that farfield attenuation is achieved in both arcs, with up to $3 \mathrm{~dB}$ more attenuation in the aft arc. The additional attenuation achieved in the forward arc is not typical of exhaust duct liner placement. This

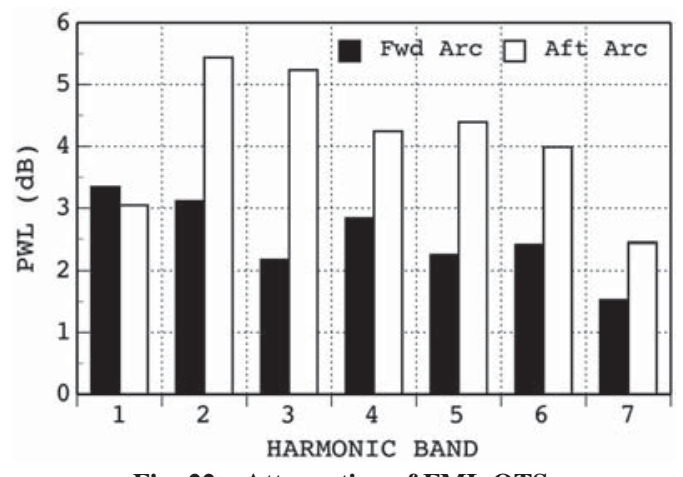

Fig. 22 Attenuation of FML OTS. bidirectionality and the increase in attenuation indicate the added benefit of placing an equal length of treatment over the source.

Figure 23 shows the attenuation vs corrected speed for the original 2 in. liner depth with a 3/32 tip gap. No significant effect due to rpm is observed.

\section{2. $\quad$ Surface Pressure Data}

Wall pressure taps were installed in the FML in the blade path region, as shown schematically in Fig. 24. A linear array of 5 taps measured the static pressure from just upstream of the leading edge to the trailing edge of the projected rotor path. Fifteen dynamic pressure transducers were flush-mounted in three staggered linear arrays of five each, also spanning the projected path. The dynamic data were acquired with the $3 / 32$ in. rotor tip gap; the static pressures were acquired with the $3 / 32$ and $1 / 32$ in. rotor tip gaps.

The time histories from the dynamic transducers were timeaveraged over 500 revolutions. Little change was seen in the dynamic response between hard-wall and FML configurations indicating that the FML presence does not affect the viscous wake effects over the rotor, and it is therefore not shown in this paper.

Wall pressures measured from the static ports are shown in Fig. 25. A modification in the static wall pressure is seen due to the presence of the liner. Ahead of the leading edge (RDP1) the wall static pressure Q6 is slightly higher with the FML, indicating a decrease in duct flow. The tip pressure rise is greater for the hard-wall configurations. This is probably due to leakage flow around the rotor tip, relieving pressure, which reduces the tip loading. Increasing the liner depth slightly increased this effect. Reducing the tip gap also reduced the pressure. 


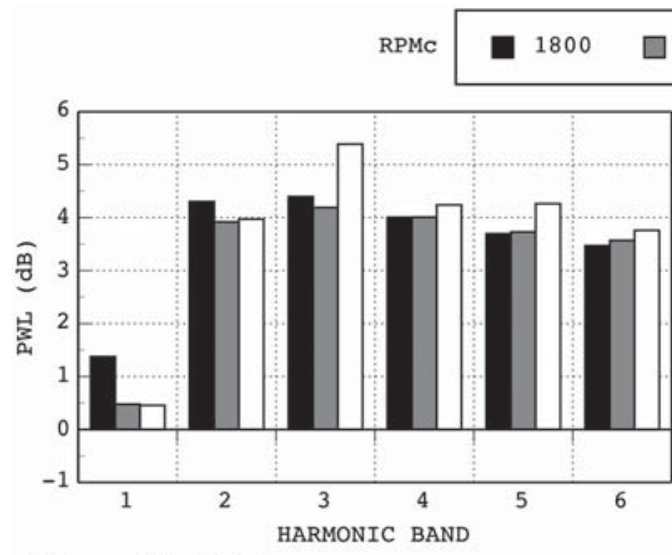

a) Forward far-field arc

\section{$1600 \square 1400$}

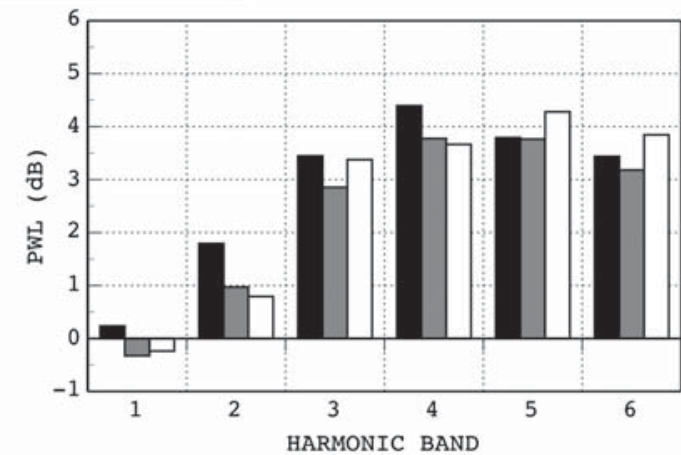

b) Aft far-field arc

Fig. 23 Effect of fan speed on FML OTR attenuation.
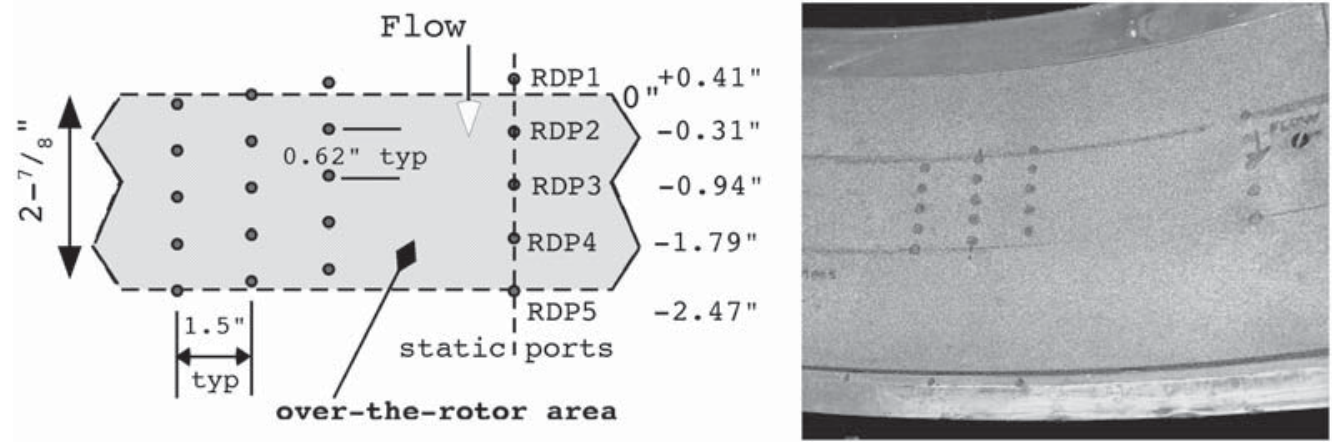

Fig. 24 Locations of static and dynamic pressure measurements in FML OTR.

\section{Flow Data}

Radial traverses of total (Kiel-type probe) and static pressure behind the rotor were acquired. The static probe traversed 1 in. behind the rotor; the total pressure traversed at $\frac{3}{4}$ and $1 \frac{1}{2}$ in. These distances are measured at the trailing edge of the rotor tip to the probe. Because the rotor untwists and the traverse remains path perpendicular to the wall, the actual distance between the rotor trailing edge and probe is reduced with immersion; the radial traverse

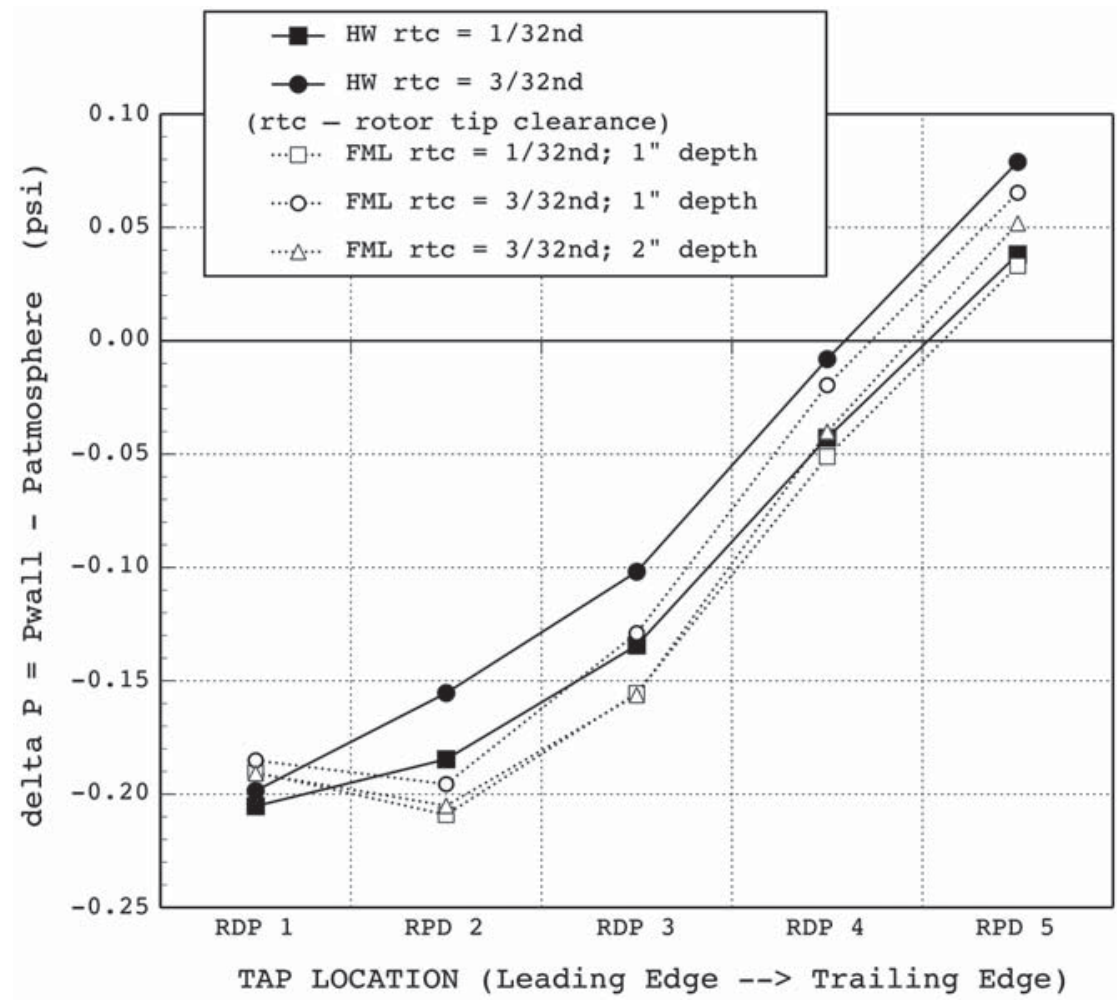

Fig. 25 Static wall pressures over the rotor (relative to atmospheric). 


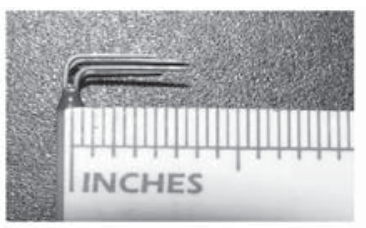

a) Hot film

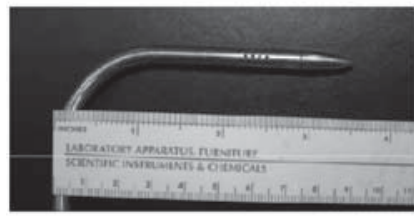

b) Static probe

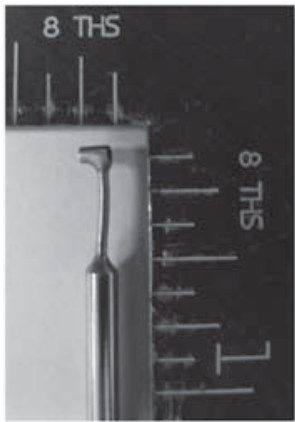

c) Kiel probe

Fig. 26 Photographs of flow measurement instrumentation and installation (TE denotes the trailing edge).

immersion was limited as a result, to prevent blade contact. The flow instrumentation is shown in Fig. 25.

Radial traverses from two hot-film types, axial/circumferential and axial/radial, were acquired for each configuration at $1800 \mathrm{rpmc}$.
Data at two axial locations were taken: $\frac{3}{4}$ and $1 \frac{1}{2}$ in. behind the rotor (measured as before, at the trailing edge of the rotor tip to the probe). Only the first $4 \mathrm{in}$. inward from the wall are presented herein, to focus on the tip effects. The passage velocity at each radial location was

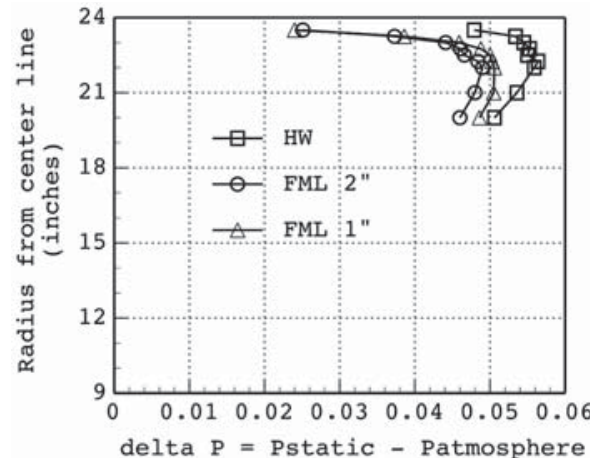

a) Static pressure behind rotor (3/32 rotor tip gap)

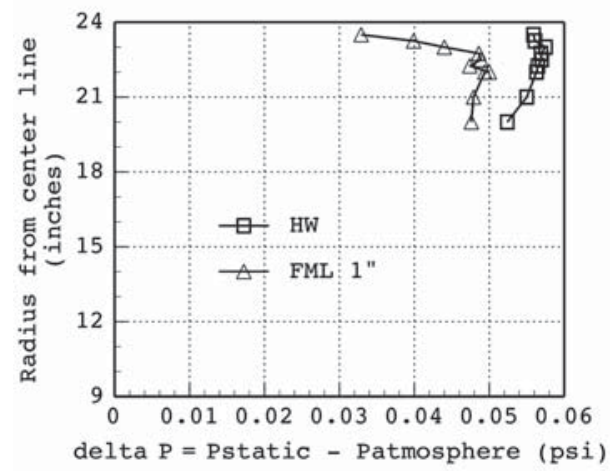

c) Static pressure behind rotor (1/32 rotor tip gap)

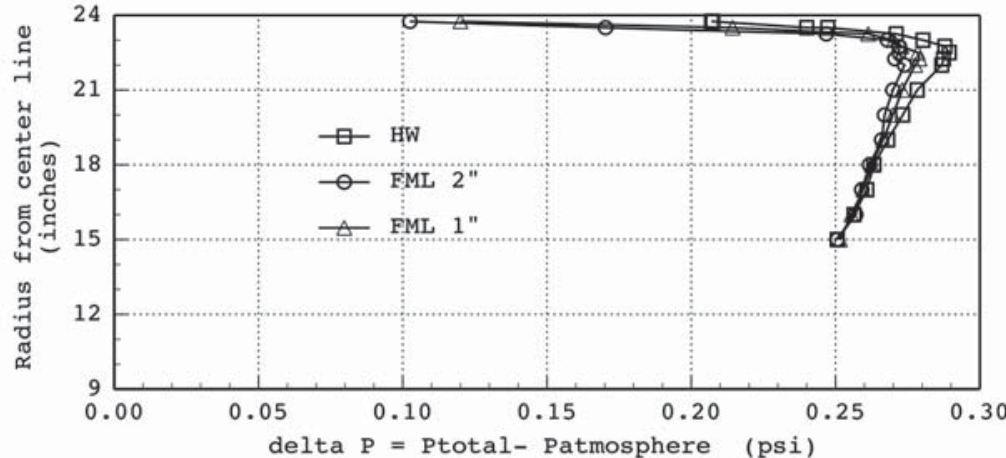

b) Total pressure behind rotor ( $3 / 32$ rotor tip gap)

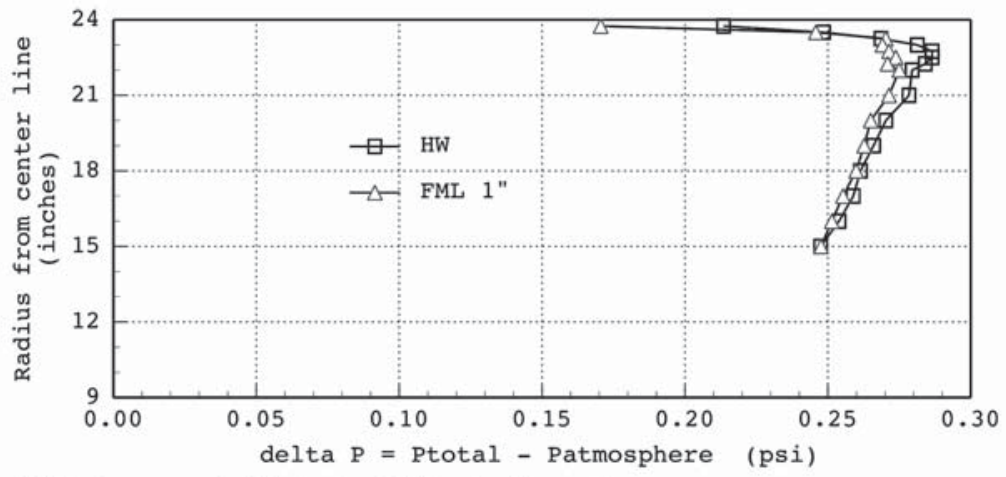

d) Total pressure behind rotor (1/32 rotor tip gap)

Fig. 27 Pitot-static pressures (relative to atmosphere) traverses behind the rotor. 


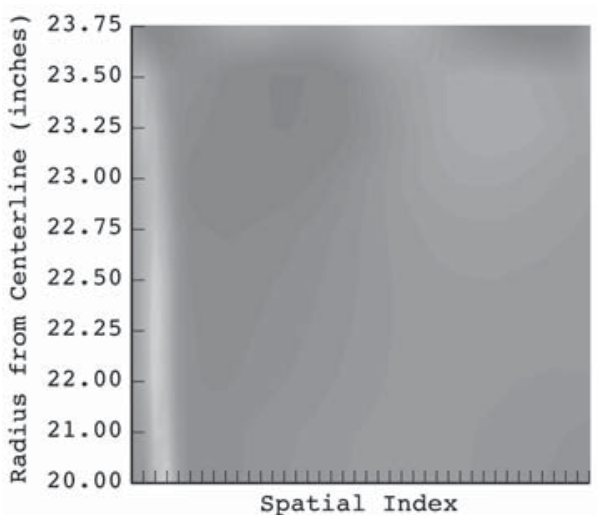

a) $\mathrm{HW}$ tip clearance $3 / 32$

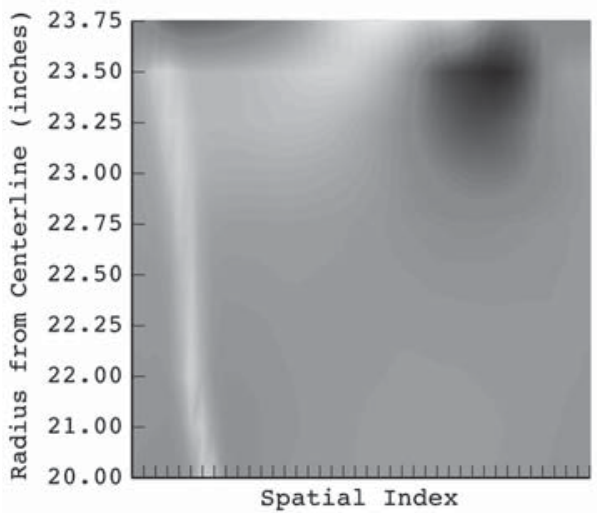

c) FML 2" tip clearance 3/32

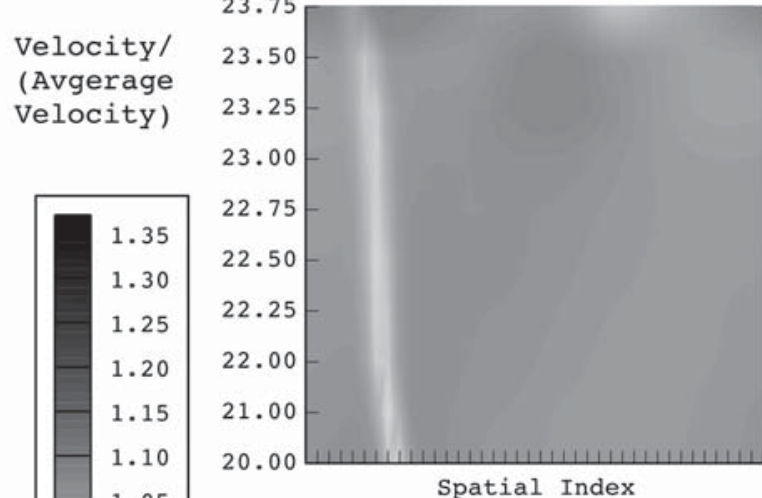

b) $\mathrm{HW}$ tip clearance $1 / 32$

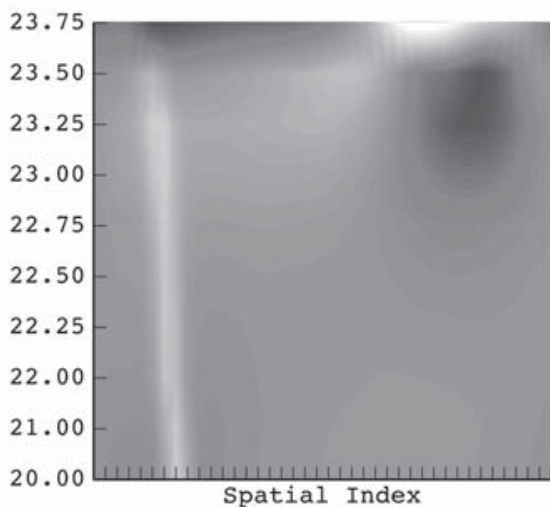

d) FML 1" tip clearance 1/32

Fig. 28 Magnitude of axial velocity ratio behind the rotor.

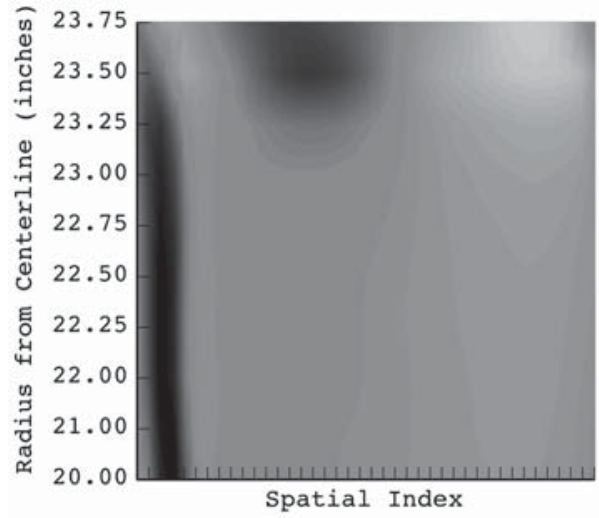

a) $\mathrm{HW}$ tip clearance $3 / 32$

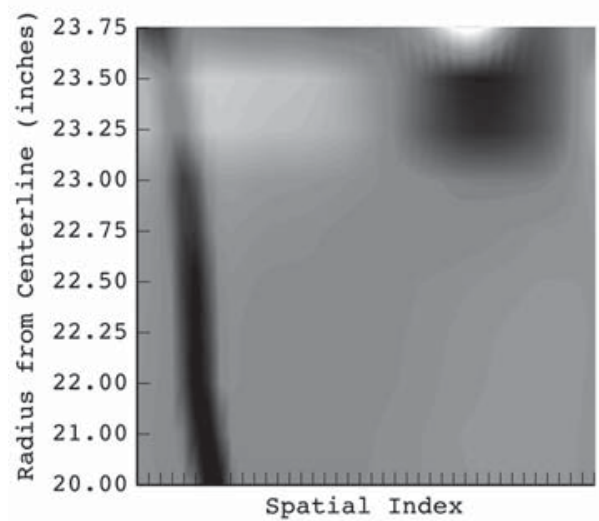

c) FML 2" tip clearance 3/32

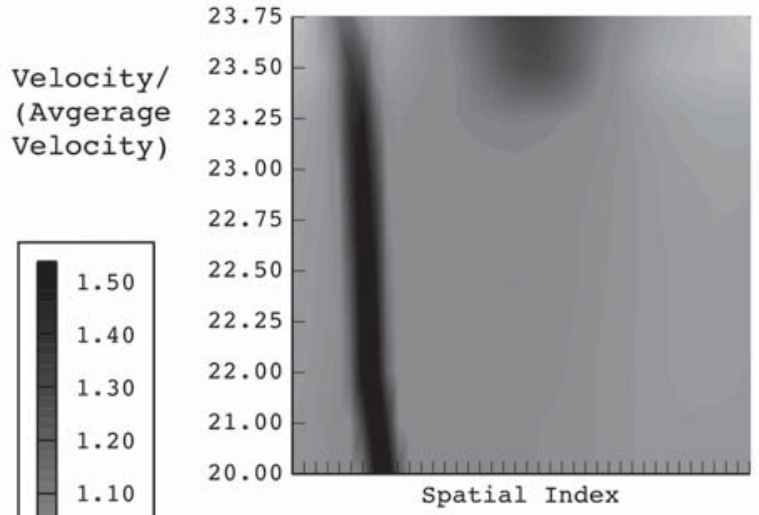

b) $\mathrm{HW}$ tip clearance $1 / 32$

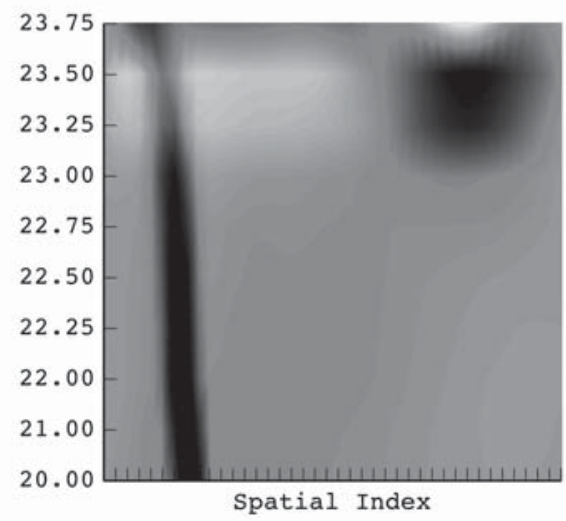

d) FML 1" tip clearance 1/32

Fig. 29 Magnitude of circumferential velocity behind the rotor. 


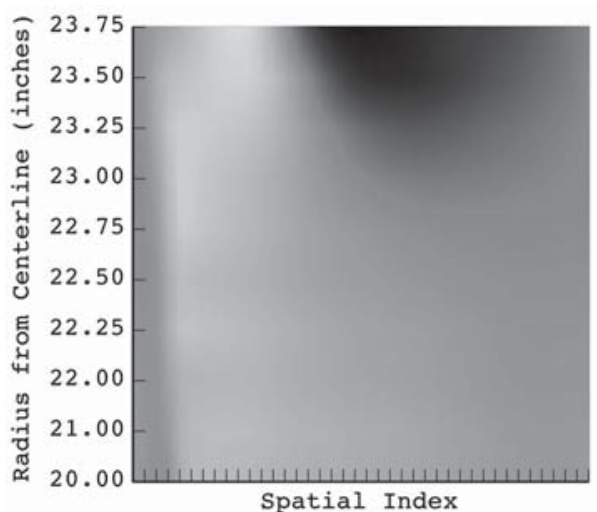

a) $\mathrm{HW}$ tip clearance $3 / 32$

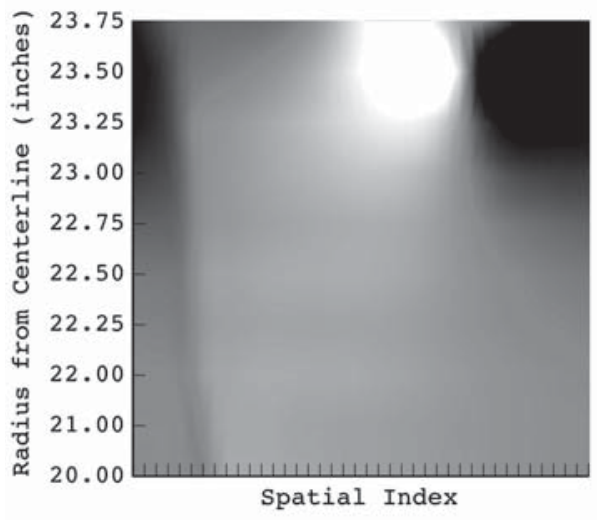

c) FML 2" tip clearance 3/32
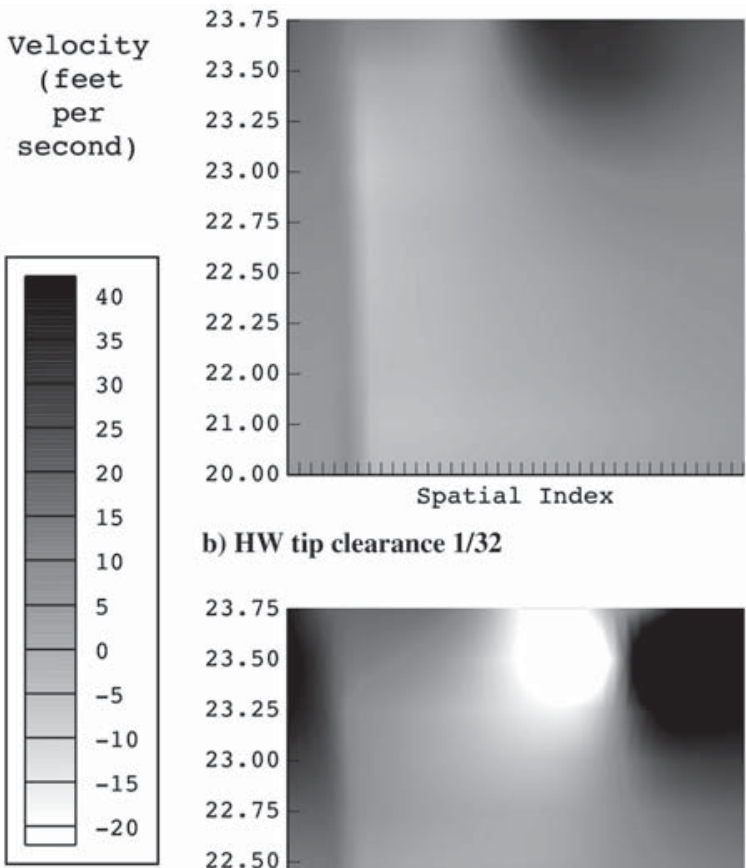

b) $\mathrm{HW}$ tip clearance $1 / 32$

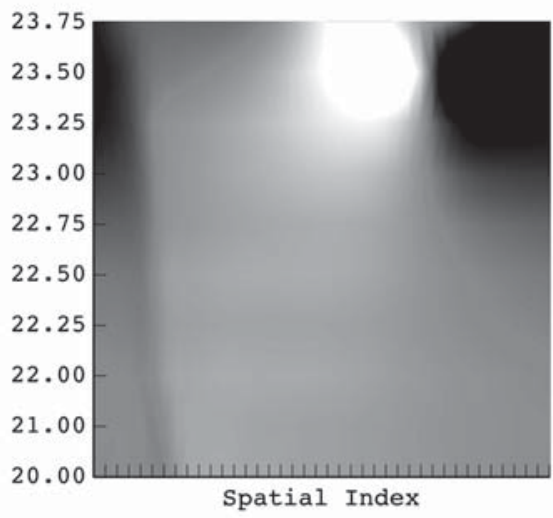

d) FML 1" tip clearance 1/32

Fig. 30 Magnitude of radial velocity behind the rotor.

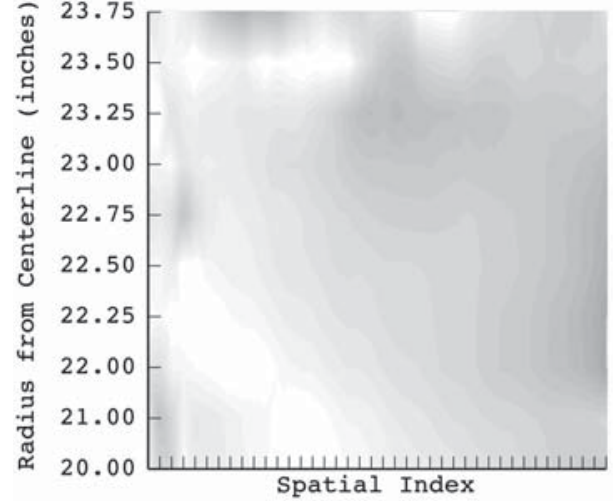

a) $\mathrm{HW}$ tip clearance $3 / 32$

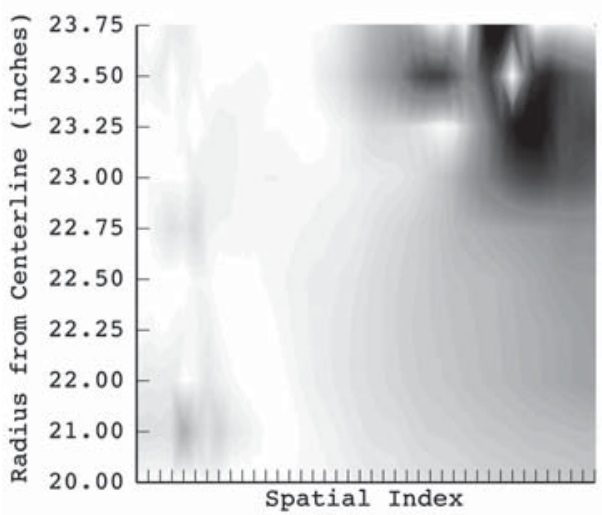

c) FML 2" tip clearance $3 / 32$

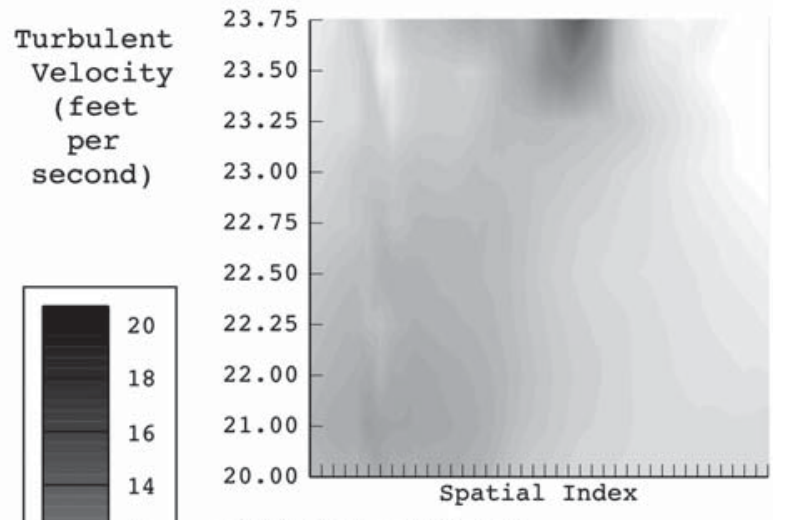

b) $\mathrm{HW}$ tip clearance $1 / 32$

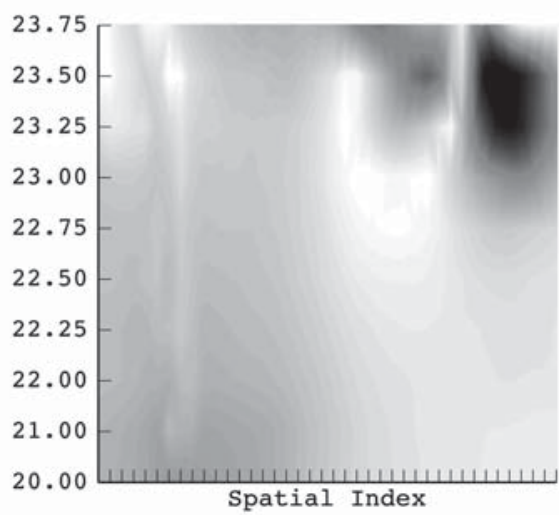

d) FML 1" tip clearance 1/32

Fig. 31 Turbulent total velocity behind the rotor. 
divided by the mean velocity at that radial location, to bring out the circumferential variations.

The pressures measured by the pressure probes (all relative to

Q7 atmosphere) are shown in Fig. 27. The liner installed over the rotor is seen to affect the pressure. A very minor difference was seen in the total pressure for a given configuration between the $\frac{3}{4}$ and $1 \frac{1}{2}$ in. radial traverses, and so pressure was interpolated to $1 \mathrm{in}$. and plotted. The presence of the FML is seen to reduce the static and total pressures; increasing the liner depth from 1 to 2 in. exacerbates this effect. Again, the suspected reason would be an increase in the tip flow resulting from the porous surface.

Figures 28-31 present two-component hot-film measurements. Figure 28 shows the axial velocity, Fig. 29 shows the circumferential velocity, and Fig. 30 shows the radial velocity behind the rotor. Figures 28a, 29a, 30a, and 31a show the hard-wall configuration with a $3 / 32$ rotor tip gap, Figs. 28b, 29b, 30b, and 31b show the hard-wall configuration with a 1/32 rotor tip gap, Figs. 28c, 29c, 30c, and 31c show the 2 -in.-deep FML configuration with a $3 / 32$ rotor tip gap, and Figs. 28d, 29d, 30d, and 31d show the 1-in.-deep FML configuration with a 1/32 rotor tip gap. Figure 31 has the same layout, except that total turbulent velocity is presented, which is obtained by subtracting the passage mean ensembles from the total velocity then calculating the rms. The axial velocity profile shows a distinct wake and weak tip vortex from the rotor in the hard-wall configuration. The presence of the FML significantly intensifies the tip vortex due to its porous nature providing a path for leakage flow. The difference in the velocity due to tip gap reduction is subtle. The stronger tip vortex increases turbulent velocity, as would be expected. It is possible that this stronger vortex could increase the rotor-stator interaction noise. This was not investigated but could be a significant concern in a highspeed fan.

\section{Conclusions}

Over-the-rotor foam-metal liners installed at or near the fan rotor provide acoustic absorption of rotor noise generated at the tips of the rotor blades and present a pressure-release boundary condition, inhibiting the rotor noise-generation source.

The acoustic characteristics of foam-metal samples were determined using a normal-incidence impedance tube. A foammetal liner was designed based on the absorption characteristics of the foam metal and the known acoustic characteristics of a low-speed fan. The attenuation characteristics of the foam-metal liner installed in the inlet matched the predicted absorption spectra reasonably well. Additional attenuation bandwidth, beyond that predicted from the impedance-tube tests, occurred with the foam-metal liner installed over the rotor.

The acoustic performance of the liner was significant, especially when placed over the rotor, achieving up to $4 \mathrm{~dB}$ of broadband attenuation in both the inlet and aft far fields. This compared favorably with the single-degree-of-freedom liners required in both the inlet and aft duct sections to achieve similar global attenuation. This would provide the opportunity to eliminate these liners, possibly shortening the ducts and reducing weight.

The foam-metal-liner effect on the flow affected the pressure near the wall in the region of the rotor and increased the size and strength of the rotor tip vortex. These measurements indicate that the attenuation observed from the foam-metal liner installed over the rotor is due to a combination of acoustic attenuation and source modification. Because of the characteristic of the low-speed, lowpressure rise, the fan's impact on thrust and efficiency cannot be effectively measured using the ANCF test bed, and therefore detailed information on these parameters was not determined. Future testing of foam-metal liners on high-speed fans should be performed and the impact on fan performance should be quantified.

\section{Acknowledgments}

The authors would like to acknowledge the foundational work of Mohan Hebsur, formerly of Ohio Aerospace Institute, who encouraged this program through his experimental investigations on the foam metal and provided the specimens for the impedance-tube testing reported in this work. Also, the high-response transducer data were acquired in collaboration with GE Corporate Research and Aviation.

\section{References}

[1] Huff, D. L., "Noise Reduction Technologies for Turbofan Engines," Proceedings of Inter-Noise 2006, IN06 732, Dec. 2006.

[2] Smith, C. D., and Parrott, T. L., "Comparison of Three Methods for Measuring Acoustic Properties of Bulk Materials," Journal of the Acoustical Society of America, Vol. 74, No. 5, 1983, pp. 1577-1582. doi:10.1121/1.390119

[3] Chung, J. Y., and Blaser, D. A., "Transfer Function Method of Measuring In-Duct Acoustic Properties. 1: Theory," Journal of the Acoustical Society of America, Vol. 68, No. 3, 1980, pp. 907-921. doi:10.1121/1.384778

[4] Jones, M. G., and Parrott, T. L., "Evaluation of a Multi-Point Method for Determining Acoustic Impedance," Mechanical Systems and Signal Processing, Vol. 3, No. 1, 1989, pp. 15-35. doi:10.1016/0888-3270(89)90020-4

[5] Lowe, R. A., Lauer, J. T., McAllister, J., and Sutliff, D. L., "The Advanced Noise Control Fan," AIAA Paper 2006-3150, 2006; also NASA TM-2006-214368, 2006.

[6] Cooper, B. A., "A Large Hemi-Anechoic Chamber Enclosure for Community-Compatible Aeroacoustic Testing of Aircraft Propulsion Systems," Journal of the Institute of Noise Control Engineering, Jan.Feb. 1994.

[7] Burdisso, R. A., and Ng, W. F., "Fan Noise Control Using HerschelQuincke Resonators," NASA CR-2003-212097, 2003.

[8] De la Riva, D. H., Burdisso, R. A., and Ng, W. F., "Aft Fan Noise Control Using Herschel/Quincke-Liner Systems," AIAA Paper 20053071, 2005. 


\section{IMPORTANT: PLEASE READ CAREFULLY.}

\section{Queries}

When production of AIAA journal papers begins, the official approved PDF is considered the authoritative manuscript. Authors are asked to submit source files that match the PDF exactly, to ensure that the final published article is the version that was reviewed and accepted by the associate editor. Once a paper has been accepted, any substantial corrections or changes must be approved by the associate editor before they can be incorporated.

If you and the EIC settled on some final changes to your manuscript after it was accepted, it is possible that your page proofs do not reflect these final changes. If that is the case, please submit these changes as itemized corrections to the proofs.

If final changes were made to the figures, please check the figures appearing in the proofs carefully. While it is usual procedure to use the figures that exist in the source file, if discrepancies are found between figures (manuscript source file vs the approved PDF), the figures from the PDF are inserted in the page proofs, again deferring to the PDF as the authoritative manuscript. If you find that agreed-upon final changes to your figures are not appearing in your page proofs, please let us know immediately.

Q1. Please check that the above copyright assignment is correct.

Q2. The tables were manually entered; please check them for accuracy.

Q3. Please supply a definition for PWL.

Q4. Figure 19 defines 6 acronyms but none of them are used in the figure. Please clarify why those acronyms are defined there or if they should all be removed. Also note that TTT is not used at all in the text.

Q5. Please adjust the text here to accommodate defining the acronyms EET, EEE, TET, and TEE

Q6. Please supply a definition for RDP1.

Q7. Please add a citation to Fig. 26 in numerical order.

Q8. The wording "fan the impact" was changed to "the fan's impact"; is that correct?

Q9. For [1], please identify IN06 732, and either a) provide the paper number and organizer's name, b) provide the page numbers and publisher's name/location (not the conference location), or c) indicate that it was a CD-ROM, who produced it, and their location.

Q10. For [6], please provide the volume number and page numbers and please check/correct the journal title, which was not found on any online databases.

Q11. HW was defined as hard wall; is that correct?

Q12. Please correct either the figure or caption, because (T) and (E) are not use in the figure.

Q13. Table cell values cannot be straddled as was done and inside borders are not permitted, and so Tables 1 and 2 were adjusted for journal style; please check that your meaning was retained. 